\title{
Twisted index theory on orbifold symmetric products and the fractional quantum Hall effect
}

\author{
Matilde Marcolli And Kyle Seipp
}

\begin{abstract}
We extend the noncommutative geometry model of the fractional quantum Hall effect, previously developed by Mathai and the first author, to orbifold symmetric products. It retains the same properties of quantization of the Hall conductance at integer multiples of the fractional Satake orbifold Euler characteristics. We show that it also allows for interesting composite fermions and anyon representations, and possibly for Laughlin type wave functions.
\end{abstract}

1 Introduction

2 Symmetric products, orbifold fundamental group, and orbifold coverings

3 Group algebras and $K$-theory

4 The magnetic field and twisted group algebras

5 Different notions of orbifold Euler characteristic

6 Higher twisted index theory

7 Orbifold braid groups and anyons

8 Laughlin-type wave functions

References 


\section{Introduction}

A satisfactory model of the integer quantum Hall effect within the framework of noncommutative geometry was developed in [9], [10]. In a 2-dimensional periodic lattice, the presence of an external magnetic field turns the classical Brillouin zone into a non-commutative torus, replacing the ordinary translational symmetries of the Hamiltonian by magnetic translations. These are symmetries of the magnetic Laplacian, and they only commute up to a phase factor, hence the appearance of the noncommutative torus as the new algebra of observables. The integer quantization of the Hall conductance can then be interpreted in terms of an index theorem on the noncommutative torus.

In [49], [50], [51] a single particle model was developed for a charged particle moving in a magnetic field within a curved geometry described by a good 2-dimensional orbifold, with the curved geometry simulating an averaged effect of the interaction with other particles. This model exhibits quantization of the Hall conductance at fractional values given by integer multiples of the Satake orbifold Euler characteristic. The results of [49], [50], [51] are based on a generalization to the (fractional) orbifold case of a previous treatment, in [14], of the integer quantum Hall effect in the hyperbolic geometry of a smooth Riemann surfaces of genus $g \geq 2$.

The main drawback of this noncommutative-geometric approach to the fractional quantum Hall effect lies in the fact that it is still based on a single particle model. While the integer quantum Hall effect is well described by an independent electron approximation, which reduces it to a single particle model, the fractional quantum Hall effect is intrinsically a many particle phenomenon: while the single particle model used in [49], [50], [51] produces a fractional quantization of the Hall conductance as a Kawasaki orbifold index-theorem on the relevant noncommutative space, it does not account for Laughlin type wave functions, nor for composite fermion (or anyon) representations.

In this paper we propose a way to extend the noncommutative geometry model of [49], [50], [51], so that it makes contact with field theories on orbifolds, of the kind considered in relation to String Theory, see e.g. [1], [21], [54], [69]. We consider systems of $n$ indistinguishable particles moving in the same type of homogeneous negatively curved geometry, under the effect of an external magnetic field, so that a classical configuration of the system is described by a point in the $n$-fold symmetric products of a good 2dimensional orbifold. As in the case of field theories on orbifolds, the relevant Fock space is given by the sum of the orbifold K-theories (or the delocalized 
equivariant cohomology) of the twisted group $C^{*}$-algebras of the wreath products $\Gamma_{n}=\Gamma^{n} \rtimes S_{n}$, with $\Gamma$ the orbifold fundamental group of the good 2-dimensional orbifold. The quantization of the Hall conductance is still obtained via a twisted higher index theorem as in [50] and is expressible in terms of the Satake orbifold Euler characteristics of the orbifold symmetric products. At the same time, the model now allows for interesting composite fermion and anyon representations, whose classification depends on Seifert invariants of orbifold line bundles. We also formulate some hypothesis, still speculative at this stage, on how to obtain Laughlin type wave functions from the geometry of the model.

The paper is structured as follows: in the rest of this introductory section we discuss the geometry of 2-dimensional good orbifolds $\Sigma$ and their symmetric products $\operatorname{Sym}^{n}(\Sigma)$. In Section 2, we introduce the relevant groups that we will be considering, related to various kinds of orbifold covers. In particular, we extend to orbifold fundamental groups a result for smooth Riemann surfaces, which identifies the (orbifold) fundamental group of the symmetric products $\operatorname{Sym}^{n}(\Sigma)$ with the abelianization of the (orbifold) fundamental group of $\Sigma$. In Section 3, we focus on K-theoretic aspects. We compute the orbifold $K$-theory of $\operatorname{Sym}^{n}(\Sigma)$, in terms of classifying spaces for proper actions and we relate it to the $K$-theory of the group $C^{*}$-algebras $C_{r}^{*}\left(\Gamma_{n}\right)$ via the Baum-Connes conjecture, which we show is satisfied by the wreath products $\Gamma_{n}$. We also discuss a possible notion of orbifold-Jacobian and its K-theoretic properties. In Section 4, we show that the magnetic field determines a compatible family of $U(1)$-multipliers $\sigma_{n}$ on $\Gamma_{n}$. We obtain in this way twisted group $C^{*}$-algebras $C_{r}^{*}\left(\Gamma_{n}, \sigma_{n}\right)$ generalizing the algebra $C_{r}^{*}(\Gamma, \sigma)$ considered in [49], [50], [51]. Using the Lyndon-Hochschild-Serre spectral sequence for the group cohomology of the wreath products, we show that the multipliers $\sigma_{n}$ define cocycles with trivial Dixmiar-Douady class. We then show that the $K$-theory of the twisted group $C^{*}$-algebra $C_{r}^{*}\left(\Gamma_{n}, \sigma_{n}\right)$ agrees with the $K$-theory of the untwisted $C_{r}^{*}\left(\Gamma_{n}\right)$. To this purpose, we prove the K-amenability of the wreath products $\mathrm{SL}(2, \mathbb{R})^{n} \rtimes S_{n}$, by adapting the argument of [30] for the K-amenability of $\mathrm{SL}(2, \mathbb{R})$. Section 5 contains expository material, where we recall and compare the different notions of orbifold Euler characteristic used in the Kawasaki index theorem and in string theory on orbifolds. The former, which we refer to as the Satake orbifold Euler characteristic is in general a rational number, while the latter, which we call the string-theoretic orbifold Euler characteristic is an integer. We recall how the latter relates to sectors and to inertia orbifolds, and how it generalizes to orbifold Chern classes. We also recall the Segal construction of the Fock space for orbifold symmetric products, based on equivariant K-theory, as 
in [64], [71]. In Section 6 we compute the twisted higher index theorem of [50] for the orbifold symmetric products $\operatorname{Sym}^{n}(\Sigma)$. Using the same relation between Hall conductance cocycle and area cocycle as in [14], [50], we show that the Hall conductance is quantized at fractional values equal to integer multiples of the Satake orbifold Euler characteristic $\chi^{\text {orb }}\left(\operatorname{Sym}^{n}(\Sigma)\right)$. In Section 7 we classify composite fermions and anyons on the symmetric products $\operatorname{Sym}^{n}(\Sigma)$. We introduce a notion of orbifold braid group, which is the orbifold fundamental group of the configuration spaces $\operatorname{Conf}(\Sigma, n)$, where the orbifold singularities are coming from the orbifold cone points of $\Sigma$. We show that, similarly to what happens in the case of Riemann surfaces and ordinary braid groups, if the orbifold $\Sigma$ has genus $g>0$, then the scalar unitary representations can only be fermions or bosons, with no non-trivial anyons. When the genus is $g=0$, there are anyon representations and we show that they are classified by the Seifert invariants of an orbifold line bundle with integer orbifold Euler number. We also show that, for arbitrary genus, there are anyon representations of higher dimensions $N$, which again depend on Seifert invariants, for an orbifold line bundle whose orbifold Euler number is in $\mathbb{Z}+(g+n-1) / N$, and with fractional statistics $\pi i / N$. Finally Section 8 contains some more speculative considerations on how to find Laughlin type wave functions in this geometric setting, in terms of the Mathai-Quillen formalism for Euler classes of vector bundles, and local systems determined by a given $N$-dimensional anyon representation. The relation between $N$ and \# $G$ imposed by the classification of anyon representations implies that one finds powers of the Vandermonde determinant with exponents equal to the denominators that appear in the quantization of the Hall conductance, as expected in Laughlin wave functions. We also suggest the possibility that Laughlin type functions may appear in computations via Selberg integrals of the orbifold Euler characteristic of a moduli space of good 2-dimensional orbifolds, analogous to the known calculations for moduli spaces of curves with marked points.

In the rest of this introductory section we review some known material that we need in the following.

\subsection{Hyperbolic 2-dimensional good orbifolds}

Let $\mathbb{H}$ denote the 2-dimensional hyperbolic plane. We will use either the upper half plane model $\mathbb{H} \cong\{z=x+i y \in \mathbb{C} \mid \Im(z)>0\}$ with the metric $d s^{2}=$ $\left(d x^{2}+d y^{2}\right) / y^{2}$ or the equivalent Poincaré disc model $\mathbb{H} \cong\{z \in \mathbb{C}|| z \mid<1\}$, with the metric $d s^{2}=4\left(d x^{2}+d y^{2}\right) /\left(1-|z|^{2}\right)^{2}$. 
On $\mathbb{H}$ we consider the isometric action of a discrete cocompact subgroup $\Gamma \subset \operatorname{PSL}(2, \mathbb{R})$, given by a Fuchsian group of signature $(g, \underline{\nu})$ with $\underline{\nu}=\left(\nu_{1}, \ldots, \nu_{m}\right)$. These groups have an explicit presentation with generators $a_{i}, b_{i}$ with $i=1, \ldots, g$ and $c_{j}$ with $j=1, \ldots, m$, of the form

$$
\Gamma=\Gamma(g, \underline{\nu})=\left\langle a_{i}, b_{i}, c_{j} \mid \prod_{i=1}^{g}\left[a_{i}, b_{i}\right] c_{1} \cdots c_{m}=1, c_{j}^{\nu_{j}}=1\right\rangle .
$$

The quotient $\Sigma=\Sigma(g, \underline{\nu})=\mathbb{H} / \Gamma$ is a 2-dimensional hyperbolic good orbifold. It is a Riemann surface of genus $g$ with $m$ cone points $\left\{x_{1}, \ldots, x_{m}\right\}$, where the point $x_{j}$ has stabilizer of order $\nu_{j}$. Any such orbifold has a finite branched covering by a smooth Riemann surface $\Sigma_{g^{\prime}}$, with $\Sigma(g, \underline{\nu})=\Sigma_{g^{\prime}} / G$ for a finite group $G$. The genus $g^{\prime}$ is related to $g$ by the Riemann-Hurwitz formula for branched coverings:

$$
g^{\prime}=1+\frac{\# G}{2}\left(2(g-1)+\left(m-\sum_{j} \nu_{j}^{-1}\right)\right) .
$$

The Riemann surface $\Sigma_{g^{\prime}}=\mathbb{H} / \Gamma^{\prime}$ has a hyperbolic uniformization by $\Gamma^{\prime} \subset$ $\operatorname{PSL}(2, \mathbb{R})$, which is related to $\Gamma(g, \underline{\nu})$ by an exact sequence

$$
1 \longrightarrow \Gamma_{g^{\prime}} \longrightarrow \Gamma(g, \underline{\nu}) \longrightarrow G \longrightarrow 1 \text {. }
$$

The Fuchsian group $\Gamma=\Gamma(g, \underline{\nu})$ is the orbifold fundamental group of the 2-dimensional orbifold $\Sigma=\Sigma(g, \underline{\nu})$, see [62].

\subsection{Symmetric products}

Let $\operatorname{Sym}^{n}(X)=X^{n} / S_{n}$, with $S_{n}$ the group of permutations of a set of $n$ elements. It is well known that, for a smooth compact Riemann surface $\Sigma$ of genus $g$, the symmetric products $\operatorname{Sym}^{n}(\Sigma)$ are smooth and are related to the Jacobian of $\Sigma$ in the following way. Let $\Omega^{1}(\Sigma)$ be the space of holomorphic 1 -forms with a basis $\left\{\omega_{1}, \ldots, \omega_{g}\right\}$. The group $\mathcal{P}_{\Sigma}$ of periods of $\Sigma$ is the subgroup $\mathcal{P}_{\Sigma} \subset \mathbb{C}^{g}$ given by the $v=\left(v_{1}, \ldots, v_{g}\right) \in \mathbb{C}^{g}$ obtained as integrals $v_{i}=\int_{\gamma} \omega_{i}$ for some $\gamma \in \pi_{1}(\Sigma)$. For a base point $x_{0} \in \Sigma$, and a path $\gamma^{\prime}$ from $x_{0}$ to $x \in \Sigma$, the integral $\int_{\gamma^{\prime}} \omega_{i}$ then defines the Abel-Jacobi map

$$
\mathcal{A}: \Sigma \rightarrow J(\Sigma)=\mathbb{C}^{g} / \mathcal{P}_{\Sigma}, \quad \mathcal{A}: x \mapsto\left(\int_{x_{0}}^{x} \omega_{1}, \ldots, \int_{x_{0}}^{x} \omega_{g}\right),
$$


where the Jacobian $J(\Sigma)$ is a torus $T^{2 g}$, which can also be identified with $H^{1}(\Sigma, \mathbb{R}) / H^{1}(\Sigma, \mathbb{Z})$. The Abel-Jacobi map extends to a map

$$
\mathcal{A}: \operatorname{Sym}^{n}(\Sigma) \rightarrow J(\Sigma), \quad \mathcal{A}:\left[x_{1}, \ldots, x_{n}\right] \mapsto \mathcal{A}\left(x_{1}\right)+\cdots+\mathcal{A}\left(x_{n}\right) .
$$

When $n>2 g-2$, the symmetric products fiber over the Jacobian with fibers that are projective spaces of dimension $n-g$,

$$
\mathbb{P}^{n-g}(\mathbb{C}) \hookrightarrow \operatorname{Sym}^{n}(\Sigma) \rightarrow J(\Sigma)
$$

Moreover, it was shown in Remark 5.8 of [35] (see also [41] for a generalization) that the fundamental group of the symmetric products of a smooth compact Riemann surface satisfies, for all $n \geq 2$,

$$
\pi_{1}\left(\operatorname{Sym}^{n}(\Sigma)\right)=\pi_{1}(\Sigma)^{a b}=H^{1}(\Sigma, \mathbb{Z})=\pi_{1}(J(\Sigma))
$$

In the following section we will consider the symmetric products $\operatorname{Sym}^{n}(\Sigma)$ of a 2-dimensional hyperbolic orbifold $\Sigma=\Sigma(g, \underline{\nu})$ and we compute the orbifold fundamental group.

\section{Symmetric products, orbifold fundamental group, and orbifold coverings}

In this section we discuss various orbifold coverings of the symmetric products $\operatorname{Sym}^{n}(\Sigma)$ and their associated groups of symmetries.

Lemma 2.1. Let $\Sigma$ be a good 2-dimensional orbifold, with singular locus $\Sigma_{\text {sing }}$ given by a finite set of cone points, and with orbifold fundamental group $\pi_{1}^{\text {orb }}(\Sigma)$. Then

$$
\pi_{1}^{o r b}\left(\Sigma^{n}\right) \cong \pi_{1}^{o r b}(\Sigma)^{n}
$$

Proof. Let $\mathcal{O}$ be a good orbifold, with singular locus of (real) codimension two, $\operatorname{codim} \mathcal{O}_{\text {sing }}=2$. Then the orbifold fundamental group $\pi_{1}^{\text {orb }}(\mathcal{O})$ of an orbifold $\mathcal{O}$ can be described $([68], \S 13)$ as the quotient

$$
\pi_{1}^{\text {orb }}(\mathcal{O})=\pi_{1}\left(\mathcal{O}_{\text {reg }}\right) / H
$$

of the fundamental group $\pi_{1}\left(\mathcal{O}_{\text {reg }}\right)$ of the regular part $\mathcal{O}_{\text {reg }}=\mathcal{O} \backslash \mathcal{O}_{\text {sing }}$ of the orbifold (the complement of the singular locus) by the normal subgroup $H$ generated by the classes $\gamma_{j}^{\nu_{j}}$ in $\pi_{1}\left(\mathcal{O}_{\text {reg }}\right)$, where $\gamma_{j}$ are loops around a 
component $\mathcal{O}_{j}$ of $\mathcal{O}_{\text {sing }}$ with $\nu_{j}$ the order of the stabilizer $G_{j}$ of $\mathcal{O}_{j}$. In particular, for a 2 -dimensional good orbifold $\Sigma$ we have $\Sigma_{\text {sing }}=\left\{x_{j}\right\}_{j=1, \ldots, m}$ the cone points with stabilizers $\mathbb{Z} / \nu_{j} \mathbb{Z}$. In the product $\Sigma^{n}$ we have $\left(\Sigma^{n}\right)_{\text {sing }}=$ $\cup_{k=1}^{n} \Sigma_{\text {sing, }(k)}$, where $\Sigma_{\text {sing, }(k)}$ means a copy of $\Sigma_{\text {sing }}$ in the $k$-th factor and the full space $\Sigma$ in all the other factors $\Sigma_{\text {sing, }(k)}=\Sigma \times \cdots \times \Sigma_{\text {sing }} \times \cdots \times \Sigma$. Thus, the set of regular points $\left(\Sigma^{n}\right)_{\text {reg }}=\Sigma^{n} \backslash \Sigma_{\text {sing }}^{n}$ is given by $\left(\Sigma^{n}\right)_{\text {reg }}=$ $\left(\Sigma_{\text {reg }}\right)^{n}$, and we can unambiguously use the notation $\Sigma_{\text {reg }}^{n}$ for this locus. We have $\pi_{1}\left(\Sigma_{\text {reg }}^{n}\right)=\pi_{1}\left(\Sigma_{\text {reg }}\right)^{n}$. The normal subgroup $H$ of $\pi_{1}\left(\Sigma_{\text {reg }}^{n}\right)$ is generated by loops $\gamma_{j, k}$ in $\Sigma^{n}$ that circle around the components $\Sigma \times \cdots \times\left\{x_{j}\right\} \times \cdots \times$ $\Sigma$ of $\Sigma_{\text {sing, } k}$. It suffices then to observe that for $k \neq k^{\prime}$ and for all $j, j^{\prime}$, the elements $\gamma_{j, k}$ and $\gamma_{j^{\prime}, k^{\prime}}$ commute in $\pi_{1}\left(\Sigma_{\text {reg }}^{n}\right)$, so that the group $H$ is a direct product $H=\prod_{k=1}^{n} H_{k}$ with each $H_{k}$ isomorphic to the normal subgroup of the $k$-th factor $\pi_{1}\left(\Sigma_{\text {reg }}\right)$, with $\pi_{1}\left(\Sigma_{\text {reg }}\right) / H_{k}=\pi_{1}^{\text {orb }}(\Sigma)$. Thus, we obtain

$$
\pi_{1}^{\text {orb }}\left(\Sigma^{n}\right)=\pi_{1}\left(\Sigma_{\mathrm{reg}}^{n}\right) / H=\pi_{1}\left(\Sigma_{\mathrm{reg}}\right)^{n} / \prod_{k} H_{k}=\pi_{1}^{\text {orb }}(\Sigma)^{n} .
$$

Let $\Gamma$ be a discrete group. Let $\Gamma^{n} \rtimes S_{n}$ be the semidirect product with multiplication

$$
\left(g_{1}, \ldots, g_{n}, \sigma\right)\left(h_{1}, \ldots, h_{n}, \tau\right)=\left(g_{1} h_{\sigma(1)}, \ldots, g_{n} h_{\sigma(n)}, \sigma \tau\right)
$$

and let $\left\langle S_{n}\right\rangle \subset \Gamma^{n} \rtimes S_{n}$ be the normal subgroup generated by the elements of $S_{n}$.

The following argument is implicit in Remark 5.8 of [35]. We reformulate it here in purely topological terms.

Lemma 2.2. Let $\Sigma$ be a good 2-dimensional orbifold, with singular locus $\Sigma_{\text {sing }}$ given by a finite set of cone points. Then the orbifold fundamental groups of the symmetric products $\operatorname{Sym}^{n}(\Sigma)$ satisfy

$$
\pi_{1}^{\text {orb }}\left(\operatorname{Sym}^{n}(\Sigma)\right) \cong \pi_{1}^{\text {orb }}(\Sigma)^{n} \rtimes S_{n} /\left\langle S_{n}\right\rangle .
$$

Proof. As in (2.2) in the previous Lemma, we have $\pi_{1}^{\text {orb }}(\Sigma)=\pi_{1}\left(\Sigma_{\mathrm{reg}}\right) / H$. The ordinary fundamental group $\pi_{1}\left(\Sigma_{\text {reg }}\right)$ classifies (ordinary) covering spaces of $\Sigma_{\text {reg }}$, in the sense that, to each normal subgroup $N$ of $\pi_{1}\left(\Sigma_{\text {reg }}\right)$ there corresponds a regular covering space $\Sigma_{N}$ of $\Sigma_{\text {reg. Such a covering }}$ space is a principal $\pi_{1}\left(\Sigma_{\text {reg }}\right) / N$-fibration over $\Sigma_{\text {reg }}$. In particular, the quotient $\pi_{1}^{\text {orb }}(\Sigma)=\pi_{1}\left(\Sigma_{\mathrm{reg}}\right) / H$ similarly classifies all such coverings of $\Sigma_{\text {reg }}$ that extend to a branched covering of $\Sigma$ with a trivial action of the stabilizers of the singular points on the corresponding fibers. Such coverings correspond to 
normal subgroups $N$ of $\pi_{1}\left(\Sigma_{\text {reg }}\right)$ that contain the normal subgroup $H$ generated by loops $\gamma_{j}$ around the components of $\Sigma_{\text {sing }}$ with the appropriate multiplicities $\nu_{j}$ as above. Next observe that a regular covering $\Sigma_{N}$ of $\Sigma_{\text {reg }}$ can also be described as an $S_{n}$-equivariant covering $\tilde{\Sigma}_{N}$ of $\Sigma_{\text {reg }}^{n} \backslash \Delta$, the complement

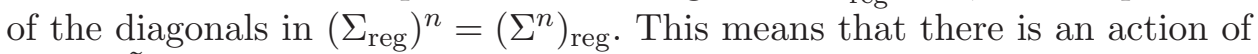
$S_{n}$ on $\tilde{\Sigma}_{N}$, which is compatible with the action on $\Sigma_{\text {reg }}^{n} \backslash \Delta$, so that the projection map of the covering is $S_{n}$-equivariant. These are classified by the crossed product $\pi_{1}\left(\Sigma_{\mathrm{reg}}^{n} \backslash \Delta\right) \rtimes S_{n}$ of the action of $S_{n}$ on the fundamental group $\pi_{1}\left(\Sigma_{\text {reg }}^{n} \backslash \Delta\right)$. Among all such coverings, we consider those that extend to an $S_{n}$-equivariant branched covering of $\Sigma^{n}$, with a trivial action of the stabilizers of the components of $\Sigma_{\text {sing }}$ and of the diagonals. These are then classified by a quotient of $\pi_{1}\left(\Sigma_{\text {reg }}^{n} \backslash \Delta\right) \rtimes S_{n}$ where we mod out by the normal subgroup generated by the loops around the components of $\Sigma_{\text {sing }}$ and the elements of $S_{n}$, that is, by the group $\pi_{1}^{\text {orb }}\left(\Sigma^{n}\right) \rtimes S_{n} /\left\langle S_{n}\right\rangle$. Finally, observe that the data of an $S_{n}$-equivariant branched covering of $\Sigma^{n}$ as above uniquely determine a branched covering over the symmetric product $\operatorname{Sym}^{n}(\Sigma)$ and vice versa, so that we can identify $\pi_{1}^{\text {orb }}\left(\Sigma^{n}\right) \rtimes S_{n} /\left\langle S_{n}\right\rangle=\pi_{1}^{\text {orb }}\left(\operatorname{Sym}^{n}(\Sigma)\right)$.

The following observation is also implicit in Remark 5.8 of [35]. We spell it out for convenience.

Lemma 2.3. Let $\Gamma^{n} \rtimes S_{n}$ be as above, with $\left\langle S_{n}\right\rangle \subset \Gamma^{n} \rtimes S_{n}$ the normal subgroup generated by the elements of $S_{n}$. There is a group isomorphism

$$
\Gamma^{n} \rtimes S_{n} /\left\langle S_{n}\right\rangle \cong \Gamma^{a b}
$$

where $\Gamma^{a b}=\Gamma /[\Gamma, \Gamma]$ is the abelianization.

Proof. Let $g_{(i)}$ denote the element $g_{(i)}=(1, \ldots, 1, g, 1, \ldots, 1)$ of $\Gamma^{n}$ that has the $i$-th coordinate equal to $g \in \Gamma$ and all the other coordinates equal to the identity element 1 . Since $\Gamma^{n}$ is a direct product of copies of $\Gamma$, the different factors $\Gamma$ commute with each other, hence for all $g, h \in \Gamma$, we have $g_{(i)} h_{(j)}=$ $h_{(j)} g_{(i)}$ whenever $i \neq j$, with the product equal to the element of $\Gamma^{n}$ with $g$ in the $i$-th place, $h$ in the $j$-th place and 1 everywhere else. Moreover, observe that, for any $g \in \Gamma$ and for $i \neq j$, the element $\left(g_{(i)}, 1\right)$ in $\Gamma^{n} \rtimes S_{n}$ is equal to $\left(1, \sigma_{i j}\right)^{-1}\left(g_{(j)}, 1\right)\left(1, \sigma_{i j}\right)$, where $\sigma_{i j} \in S_{n}$ is the permutation that exchanges $i$ and $j$ and fixes all other elements of $\{1, \ldots, n\}$. For any $\gamma \in \Gamma^{n} \rtimes S_{n}$, and for all $\sigma \in S_{n}$, we have $(1, \sigma)^{-1} \gamma(1, \sigma) \gamma^{-1} \in\left\langle S_{n}\right\rangle$, hence $\gamma$ and $(1, \sigma)^{-1} \gamma(1, \sigma)$ define the same class in the quotient $\Gamma^{n} \rtimes S_{n} /\left\langle S_{n}\right\rangle$. In particular, $g_{(i)}$ and $g_{(j)}$ define the same element in the quotient, for all $g \in \Gamma$ and for all $i \neq j$. Thus, we obtain that in the quotient the $n$-copies of $\Gamma$ in the product $\Gamma^{n}$ 
are identified and commutators are killed, hence the quotient gets identified with $\Gamma^{a b}$.

Combining the results of Lemmata 2.1, 2.2, and 2.3, we obtain the analog of (1.7) for orbifold fundamental groups.

Proposition 2.4. For $n \geq 2$, the symmetric products $\operatorname{Sym}^{n}(\Sigma)$ of a good 2-dimensional orbifold $\Sigma$ have orbifold fundamental group given by

$$
\pi_{1}^{\text {orb }}\left(\operatorname{Sym}^{n}(\Sigma)\right) \cong \pi_{1}^{\text {orb }}(\Sigma)^{a b}
$$

In the case of a 2-dimensional hyperbolic orbifold $\Sigma=\Sigma(g, \underline{\nu})$ one obtains the following.

Corollary 2.5. For $\Sigma=\Sigma(g, \underline{\nu})$ and $n \geq 2$, we have $\pi_{1}^{\text {orb }}\left(\operatorname{Sym}^{n}(\Sigma(g, \underline{\nu}))\right)=$ $\mathbb{Z}^{2 g} \oplus \mathbb{Z}_{\nu}$, where $\mathbb{Z}_{\nu}=\oplus_{j=1}^{m} \mathbb{Z} / \nu_{j} \mathbb{Z}$.

Proof. The abelianization of a group $\Gamma=\Gamma(g, \underline{\nu})$ of the form (1.1) is given by $\mathbb{Z}^{2 g} \oplus_{j} \mathbb{Z} / \nu_{j} \mathbb{Z}$.

Remark 2.6. The abelianization of the fundamental group is the first homology group, $\pi_{1}(\Sigma)^{a b}=H^{1}(\Sigma, \mathbb{Z})$. In the case of the orbifold fundamental group, there is a similar homological interpretation of its abelianization, in terms of the $t$-singular homology defined in $[65], \pi_{1}^{\text {orb }}(\Sigma)^{a b}=t H^{1}(\Sigma, \mathbb{Z})$, where the $t$-singular homology $t H^{*}$ is constructed using singular simplexes that intersect transversely the singular locus of $\Sigma$, see [65] for details.

\subsection{Geometry of some orbifold covering spaces}

We consider some covering spaces of the orbifold symmetric products that will be useful in the rest of the paper.

Proposition 2.7. For $n \geq 2$, let $\operatorname{Sym}^{n}(\Sigma)$ be the symmetric product of the 2-dimensional orbifold $\Sigma=\Sigma(g, \underline{\nu})$. Let $\left\langle S_{n}\right\rangle$ be the normalizer of $S_{n}$ in $\Gamma^{n} \rtimes S_{n}$ and let $\mathbb{S}_{n}=\left\langle S_{n}\right\rangle / S_{n}$. Let $\mathbb{S}^{n}(\mathbb{H}):=\mathbb{H}^{n} /\left\langle S_{n}\right\rangle$. Let $\Sigma_{g^{\prime}}=\mathbb{H} / \Gamma_{g^{\prime}}$ with $\Gamma_{g^{\prime}}$ as in (1.3) with finite quotient $G=\Gamma / \Gamma_{g^{\prime}}$. Let $G_{n}:=G^{n} \rtimes S_{n}$.

1) $\operatorname{Sym}^{n}(\Sigma)$ is orbifold covered by $\mathbb{H}^{n}$, with $\operatorname{Sym}^{n}(\Sigma)=\mathbb{H}^{n} / \Gamma^{n} \rtimes S_{n}$.

2) $\operatorname{Sym}^{n}(\Sigma)$ is orbifold covered by $\mathbb{S}^{n}(\mathbb{H})$, with $\operatorname{Sym}^{n}(\Sigma)=\mathbb{S}^{n}(\mathbb{H}) / \pi_{1}^{\text {orb }}\left(\operatorname{Sym}^{n}(\Sigma)\right)$.

3) $\mathbb{S}^{n}(\mathbb{H})$ is orbifold covered by $D^{2 n}=\operatorname{Sym}^{n}(\mathbb{H})$, with $\mathbb{S}^{n}(\mathbb{H})=D^{2 n} / \mathbb{S}_{n}$. 
4) $\operatorname{Sym}^{n}(\Sigma)$ is orbifold covered by the smooth manifold $\Sigma_{g^{\prime}}^{n}$, with $\operatorname{Sym}^{n}(\Sigma)=\Sigma_{g^{\prime}}^{n} / G_{n}$.

Proof. The orbifold $\Sigma=\Sigma(g, \underline{\nu})$ has a finite branched cover by a smooth surface $\Sigma_{g^{\prime}}$, of genus (1.2), so that $\Sigma=\Sigma_{g^{\prime}} / G$, with the finite group $G$ as in (1.3). Moreover, $\Sigma$ also has universal orbifold cover $\mathbb{H}$, with $\Sigma=\mathbb{H} / \Gamma$, for $\Gamma=\Gamma(g, \underline{\nu})$. The isometric action of $\Gamma$ on $\mathbb{H}$ induces an isometric action of $\Gamma^{n} \rtimes S_{n}$ on the $n$-fold product $\mathbb{H}^{n}$, with quotient $\mathbb{H}^{n} / \Gamma^{n} \rtimes S_{n}=\operatorname{Sym}^{n}(\Sigma)$. Consider the normal subgroup $\left\langle S_{n}\right\rangle \subset \Gamma^{n} \rtimes S_{n}$. We can equivalently describe the quotient above as $\left(\mathbb{H}^{n} /\left\langle S_{n}\right\rangle\right) /\left(\Gamma^{n} \rtimes S_{n} /\left\langle S_{n}\right\rangle\right)=\operatorname{Sym}^{n}(\Sigma)$. The group $S_{n}$ is normal inside $\left\langle S_{n}\right\rangle$ with quotient $\mathbb{S}_{n}$, and we can further write the quotient $\mathbb{H}^{n} /\left\langle S_{n}\right\rangle=\left(\mathbb{H}^{n} / S_{n}\right) / \mathbb{S}_{n}=\operatorname{Sym}^{n}(\mathbb{H}) / \mathbb{S}_{n}$. We identify the hyperbolic plane $\mathbb{H}$ with its Poincaré disc model $\mathbb{H}=D^{2}$ (the open unit disc in $\mathbb{R}^{2}$ with the hyperbolic metric). By Lemma 5 of [42], there is a homeomorphism of pairs

$$
\left(D^{2 n}, \partial D^{2 n}=S^{2 n-1}\right) \cong\left(\operatorname{Sym}^{n}\left(D^{2}\right), \operatorname{Sym}^{n}\left(\overline{D^{2}}\right) \backslash \operatorname{Sym}^{n}\left(D^{2}\right)\right),
$$

where $D^{2 n}$ is an open $2 n$-dimensional disc. Thus, we can identify $\operatorname{Sym}^{n}(\mathbb{H}) \cong$ $D^{2 n}$, with the metric induced by the hyperbolic metric on $\mathbb{H}$, so that $\operatorname{Sym}^{n}(\mathbb{H}) / \mathbb{S}_{n}=D^{2 n} / \mathbb{S}_{n}$. Finally, consider the sequence of groups (1.3). The normal embedding $\Gamma_{g^{\prime}} \hookrightarrow \Gamma$ determines a normal embedding $\Gamma_{g^{\prime}}^{n} \hookrightarrow \Gamma^{n} \rtimes$ $S_{n}$. The quotient group can be identified with $G^{n} \rtimes S_{n}$, where $G=\Gamma / \Gamma_{g^{\prime}}$. We then rewrite the quotient $\mathbb{H}^{n} / \Gamma^{n} \rtimes S_{n}=\operatorname{Sym}^{n}(\Sigma)$ as $\left(\mathbb{H}^{n} / \Gamma_{g^{\prime}}^{n}\right) /\left(\Gamma^{n} \rtimes\right.$ $\left.S_{n} / \Gamma_{g^{\prime}}^{n}\right)=\Sigma_{g^{\prime}}^{n} / G_{n}$, with the finite group $G_{n}=G^{n} \rtimes S_{n}$.

\section{Group algebras and $K$-theory}

We now compute the orbifold K-theory groups of the symmetric products $\operatorname{Sym}^{n}(\Sigma)$ and we discuss their relation to the K-theory of group $C^{*}$-algebras.

\subsection{Orbifold $K$-theory}

Let $X$ be a good orbifold that is orbifold covered by a smooth manifold $Y$ with $X=Y / G$. Then the orbifold $K$-theory of $X$ is given by

$$
K_{\text {orb }}^{\bullet}(X)=K_{\bullet}\left(C_{0}(Y) \rtimes G\right)=K_{G}^{\bullet}(Y),
$$

the $G$-equivariant $K$-theory of $Y$. 
We know from [26], [49] that, for a good 2-dimensional orbifold $\Sigma=$ $\Sigma(g, \underline{\nu})$, with $m$ cone points $x_{j}$ with stabilizers of order $\nu_{j}$, the orbifold $K$ theory is given by

$$
K_{\text {orb }}^{\bullet}(\Sigma)=\left\{\begin{array}{ll}
\mathbb{Z}^{2-m+\nu} & \bullet=0 \\
\mathbb{Z}^{2 g} & \bullet=1
\end{array},\right.
$$

where $\nu=\sum_{j=1}^{m} \nu_{j}$.

For the symmetric products $\operatorname{Sym}^{n}(\Sigma)$, using the covering (4) of Proposition 2.7 , we obtain

$$
K_{\text {orb }}^{\bullet}\left(\operatorname{Sym}^{n}(\Sigma)\right)=K_{\bullet}\left(\left(C\left(\Sigma_{g^{\prime}}\right) \rtimes G\right)^{\otimes n} \rtimes S_{n}\right)=K_{G^{n} \rtimes S_{n}}^{\bullet}\left(\Sigma_{g^{\prime}}^{n}\right),
$$

where $\left.K_{\bullet}\left(C\left(\Sigma_{g^{\prime}}\right) \rtimes G\right)\right)=K_{\text {orb }}^{\bullet}(\Sigma)$. Using the orbifold cover (1) of Proposition 2.7 , we see that it can also be described as

$$
K_{\text {orb }}^{\bullet}\left(\operatorname{Sym}^{n}(\Sigma)\right)=K_{\Gamma^{n} \rtimes S_{n}}^{\bullet}\left(\mathbb{H}^{n}\right) .
$$

These descriptions of the orbifold $K$-theory of the symmetric products fall into a general framework for studying equivariant $K$-theory with respect to the action on powers $X^{n}$ of the wreath products

$$
G \sim S_{n}:=G^{n} \rtimes S_{n},
$$

for a finite group $G$ acting on a smooth manifold $X$. Several important properties of the equivariant $K$-theory groups $K_{G^{n} \rtimes S_{n}}^{\bullet}\left(X^{n}\right)$ where studied in [71]. We recall some of the main results of [71] and we apply them to our case, described as in (3.3).

\subsection{Classifying space for proper action and assembly map}

It is known from [6] that, to a locally compact group $G$ one can associate a universal space for proper actions $\underline{E} G$, and a classifying space for proper actions given by the quotient $\underline{B} G=\underline{E} G / G$, so that there is a Kasparov assembly map from the equivariant $K$-homology groups with $G$-compact support $K_{\bullet}^{G}(\underline{E} G)$ to the $K$-theory of the reduced group $C^{*}$-algebra

$$
\mu: K_{j}^{G}(\underline{E} G) \rightarrow K_{j}\left(C_{r}^{*}(G)\right),
$$

which assigns to an abstract $G$-equivariant elliptic operator its index. The group $G$ satisfies the Baum-Connes conjecture if the map (3.6) is an isomorphism. 
The Baum-Connes conjecture (in fact the stronger form with coefficients) is implied by the Haagerup property, [53]. All finite groups satisfy the Haagerup property, and Fuchsian groups are also in the list of groups that are known to satisfy it, see [15], [53]. However, while it is known from [18] that the class of groups satisfying the Haagerup property is closed under wreath products, this only refers to "standard" wreath products $G \sim H:=G^{(H)} \rtimes H$ where $G^{(H)}=\oplus_{h \in H} G$. A more general class of wreath products, which includes the case $G^{n} \rtimes S_{n}$ that we are interested in, is given by the "permutation wreath products" $G \sim_{X} H=G^{(X)} \rtimes H$, where $X$ is an $H$-set and the action of $H$ on $G^{(X)}=\oplus_{x \in X} G$ is by permuting indices $x \in X$ with the $H$ action. As shown in [18], the Haagerup property is a lot more delicate for the case of the permutation wreath products.

However, for two groups $G$ and $H$ that both satisfy the Haagerup property, even if the more general permutation wreath products $G \sim_{X} H=$ $G^{(X)} \rtimes H$ do not necessarily satisfy the Haagerup property, they all do satisfy the Baum-Connes conjecture. This follows from the general result of Oyono-Oyono on Baum-Connes for certain group extensions, [58]. Thus, we have the following property.

Lemma 3.1. The groups $\Gamma^{n} \rtimes S_{n}$, with $\Gamma=\Gamma(g, \underline{\nu})$ a Fuchsian group, satisfy the Baum-Connes conjecture, hence the assembly map

$$
\mu: K_{\text {orb }}^{\bullet}\left(\operatorname{Sym}^{n}(\Sigma)\right)=K_{\Gamma^{n} \rtimes S_{n}}^{\bullet}\left(\mathbb{H}^{n}\right) \rightarrow K_{\bullet}\left(C_{r}^{*}\left(\Gamma^{n} \rtimes S_{n}\right)\right)
$$

is an isomorphism.

Proof. The general result of [58] implies that the wreath products $\Gamma^{n} \rtimes S_{n}$ satisfy the Baum-Connes conjecture. The result then follows by identifying $\mathbb{H}^{n}=\underline{E}\left(\Gamma^{n} \rtimes S_{n}\right)$ and $\operatorname{Sym}^{n}(\Sigma)=\underline{B}\left(\Gamma^{n} \rtimes S_{n}\right)$, with models for the universal and classifying space for proper actions, respectively. To see this, we can use the fact that if for a group $G$ a $G$-space $Y$ is a model of the universal space for proper actions $\underline{E} G$, and $G^{\prime} \subset G$ is a subgroup, then $Y$ is also a model of $E G^{\prime}$ (Corollary 1.9 of [6]) and that if $G$ is a Lie group and $K$ is the maximal compact subgroup, then a model of $\underline{E} G$ is given by the quotient $G / K$. A Lie group is virtually connected if it has only finitely many connected components. For any virtually connected Lie group the quotient $G / K$ is diffeomorphic to a Euclidean space. We apply the above to the group $\operatorname{PSL}(2, \mathbb{R})^{n} \rtimes S_{n}$. 


\subsection{Orbifold $K$-theory and Lie group quotient}

Consider as above the virtually connected Lie group $\operatorname{PSL}(2, \mathbb{R})^{n} \rtimes S_{n}$ and let $\mathcal{K}_{n}$ denote its maximal compact subgroup, with quotient $\operatorname{PSL}(2, \mathbb{R})^{n} \rtimes$ $S_{n} / \mathcal{K}_{n} \simeq \mathbb{H}^{n}$. The orbifold symmetric product is obtained as the double quotient

$$
\operatorname{Sym}^{n}(\Sigma)=\Gamma_{n} \backslash \operatorname{PSL}(2, \mathbb{R})^{n} \rtimes S_{n} / \mathcal{K}_{n},
$$

where $\Gamma_{n}=\Gamma^{n} \rtimes S_{n}$. Let

$$
\begin{aligned}
& \mathcal{P}_{n}:=\Gamma_{n} \backslash \operatorname{PSL}(2, \mathbb{R})^{n} \rtimes S_{n}, \\
& \hat{\mathcal{P}}_{n}:=\Gamma_{g^{\prime}}^{n} \backslash \operatorname{PSL}(2, \mathbb{R})^{n} \rtimes S_{n} .
\end{aligned}
$$

Then we have the following.

Lemma 3.2. Let $\mathcal{P}_{n}, \hat{\mathcal{P}}_{n}$, and $\mathcal{K}_{n}$ be as above and let $G_{n}=G^{n} \rtimes S_{n}$, with $G=\Gamma / \Gamma_{g^{\prime}}$. The algebras $C_{0}\left(\mathcal{P}_{n}\right) \rtimes \mathcal{K}_{n}$ and $C_{0}\left(\hat{\mathcal{P}}_{n} / \mathcal{K}_{n}\right) \rtimes G_{n}=C\left(\Sigma_{g^{\prime}}^{n}\right) \rtimes G_{n}$ are strongly Morita equivalent.

Proof. By (3.8) and (3.9), and the fact that $\Gamma_{g^{\prime}}^{n} \subset \Gamma^{n} \rtimes S_{n}$ is a normal subgroup with quotient $G_{n}=G^{n} \rtimes S_{n}$, we obtain

$$
\mathcal{P}_{n}=G_{n} \backslash \hat{\mathcal{P}}_{n}, \quad \text { and } \quad \hat{\mathcal{P}}_{n} / \mathcal{K}_{n}=\Gamma_{g^{\prime}}^{n} \backslash \mathbb{H}^{n}=\Sigma_{g^{\prime}}^{n}
$$

The Morita equivalence then follows as in Proposition 1.2 of [49], by applying $[34]$.

The orbifold $C^{*}$-algebra is defined in [26] as

$$
C^{*}\left(\operatorname{Sym}^{n}(\Sigma)\right)=C\left(\mathcal{F}_{n}\right) \rtimes S O(2 n),
$$

where $\mathcal{F}_{n}$ is the frame bundle of the orbifold tangent bundle of $\operatorname{Sym}^{n}(\Sigma)$. By the same argument of [26] it is shown to be strongly Morita equivalent to

$$
C^{*}\left(\operatorname{Sym}^{n}(\Sigma)\right) \simeq C\left(\Sigma_{g^{\prime}}^{n}\right) \rtimes G_{n} .
$$

Thus, combining [26] with Proposition 2.7, we obtain the following.

Corollary 3.3. The algebras $C^{*}\left(\operatorname{Sym}^{n}(\Sigma)\right), C\left(\Sigma_{g^{\prime}}^{n}\right) \rtimes G_{n}, C_{0}\left(\mathbb{H}^{n}\right) \rtimes \Gamma_{n}$, $C_{0}\left(\mathbb{S}^{n}(\mathbb{H})\right) \rtimes \Gamma^{a b}$, and $C_{0}\left(\mathcal{P}_{n}\right) \rtimes \mathcal{K}_{n}$ are all strongly Morita equivalent. 


\subsection{A notion of orbifold-Jacobian}

Given a good 2-dimensional orbifold $\Sigma=\Sigma(g, \underline{\nu})$, we define the orbifoldJacobian of $\Sigma$ to be the product

$$
J^{o r b}(\Sigma):=J(\Sigma) \times \prod_{j=1}^{m} \mu_{\nu_{j}}
$$

where $J(\Sigma)=H^{1}(\Sigma, \mathbb{R}) / H^{1}(\Sigma, \mathbb{Z})=\mathbb{T}^{2 g}$, a real torus of rank $2 g$, and $\mu_{\nu_{j}}$ denotes the group of roots of unity of order $\nu_{j}$. The group structure on $J^{o r b}(\Sigma)$ is the direct product $\mathbb{T}^{2 g} \times \prod_{j=1}^{m} \mu_{\nu_{j}}$.

For each cone point $x_{j}, j=1, \ldots, m$ on the orbifold $\Sigma$, let $C_{j}$ be the boundary of a small disc in $\Sigma$ centered at $x_{j}$. Let $x_{j, k}$ for $k=1, \ldots, \nu_{j}$ be points on $C_{j}$. For such a collection of base points, we define an orbifoldAbel-Jacobi map $\mathcal{A}^{\text {orb }}=\left\{\mathcal{A}_{j, k}\right\}$ with

$$
\mathcal{A}_{j, k}: \Sigma \rightarrow \mathbb{T}^{2 g} \times\left\{\zeta_{j, k}\right\}, \quad \mathcal{A}_{j, k}: \omega \mapsto \int_{x_{j, k}}^{x} \omega
$$

where $\zeta_{j, k}$ are the roots of unity in $\mu_{\nu_{j}}$. This extends to an orbifold-AbelJacobi map $\mathcal{A}^{\text {orb }}: \operatorname{Sym}^{n}(\Sigma) \rightarrow J^{\text {orb }}(\Sigma)$ by $\mathcal{A}^{\text {orb }}\left[x_{1}, \ldots, x_{n}\right]=\mathcal{A}^{\text {orb }}\left(x_{1}\right)+\cdots+$ $\mathcal{A}^{\text {orb }}\left(x_{n}\right)$.

\section{5. $K$-theory and the orbifold-Jacobian}

The reduced group $C^{*}$-algebra $C_{r}^{*}(G)$ of a discrete group $G$ is the norm closure in the algebra of bounded operators on $\ell^{2}(G)$ of the group ring $\mathbb{C}[G]$, acting via the left regular representation $L_{g} \xi\left(g^{\prime}\right)=\xi\left(g^{-1} g^{\prime}\right)$.

Lemma 3.4. The reduced group $C^{*}$-algebra $C_{r}^{*}\left(\pi_{1}^{\text {orb }}\left(\operatorname{Sym}^{n}(\Sigma)\right)\right)$ has $K$ theory isomorphic to the topological K-theory of the orbifold-Jacobian $J^{o r b}(\Sigma)$.

Proof. The group $\pi_{1}^{\text {orb }}\left(\operatorname{Sym}^{n}(\Sigma)\right)=\pi_{1}^{\text {orb }}(\Sigma)^{a b}$ is abelian. Thus, the $K$-theory of the reduced group $C^{*}$-algebra can be identified with the topological $K$ theory of the dual group, under Pontrjagin duality,

$$
K_{j}\left(C_{r}^{*}\left(\pi_{1}^{o r b}(\Sigma)^{a b}\right)\right) \simeq K_{j}\left(C\left(\pi_{1}^{o r b}(\Sigma)^{a b}\right)\right) .
$$

We have $\pi_{1}^{\text {orb }}(\Sigma)^{a b}=\mathbb{Z}^{2 g} \oplus \bigoplus_{j} \mathbb{Z} / \nu_{j} \mathbb{Z}$. The Pontrjagin dual of a direct sum of abelian groups is the direct product of the Pontrjagin duals. The dual of 
$\mathbb{Z}^{2 g}$ is a $2 g$-dimensional real torus $\mathbb{T}^{2 g}=S^{1} \times \cdots \times S^{1}$, while for each finite group $\mathbb{Z} / \nu_{j} \mathbb{Z}$ the Pontrjagin dual is the subgroup $\mu_{\nu_{j}} \subset S^{1}$ of $\nu_{j}$-th roots of unity, which can be identified again with $\mathbb{Z} / \nu_{j} \mathbb{Z}$. Thus, we obtain a direct product

$$
\widehat{\pi_{1}^{o r b}(\Sigma)^{a b}}=\mathbb{T}^{2 g} \times \prod_{j} \mu_{\nu_{j}}
$$

\subsection{A homotopy theoretic version}

Consider a smooth surface $\Sigma_{g^{\prime}}=\mathbb{H} / \Gamma_{g^{\prime}}$ uniformized by the hyperbolic plane $\mathbb{H}$. The surface $\Sigma_{g^{\prime}}$ is a model of the classifying space for proper actions $\Sigma_{g^{\prime}}=\underline{B} \Gamma_{g^{\prime}}$, with $\mathbb{H}=\underline{E} \Gamma_{g^{\prime}}$ a model for a universal space for proper actions, [6].

It is known (see Theorem 1.1 of [70]) that a group homomorphism $\alpha$ : $\Gamma_{1} \rightarrow \Gamma_{2}$ induces a commutative diagram

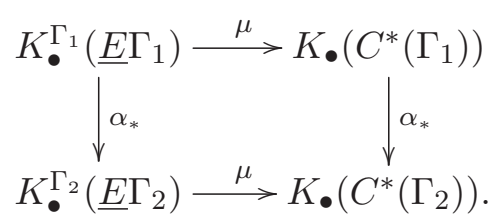

The analogous statement for reduced algebras $C_{r}^{*}\left(\Gamma_{i}\right)$ holds in general only for monomorphisms (Corollary 1.2 of [70]), but since the geometric lefthand-side is always functorial, under the hypothesis that the Baum-Connes conjecture holds, then the right-hand-side would also be functorial for the reduced case, as observed in [70]. We focus on the case where $\Gamma_{1}=\Gamma_{g^{\prime}}$ and $\Gamma_{2}=\Gamma_{g^{\prime}}^{a b}=H^{1}\left(\Sigma_{g^{\prime}}, \mathbb{Z}\right)$, with $\alpha: \Gamma_{g^{\prime}} \rightarrow \Gamma_{g^{\prime}}^{a b}$ the quotient map. In this case, we know that the groups involved satisfy the Baum-Connes conjecture, and we can think of the left-hand-side of the diagram (3.12) as a kind of "homotopytheoretic Abel-Jacobi map" from the $K$-homology of the curve $\Sigma_{g^{\prime}}$ to that if its Jacobian,

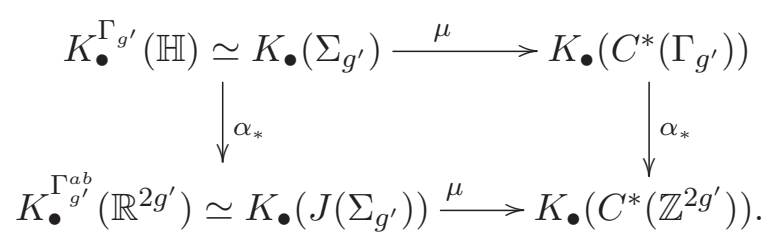

In a similar way, we obtain maps

$$
K_{\bullet}^{\Gamma}(\mathbb{H}) \simeq K_{\bullet}^{\text {orb }}(\Sigma) \stackrel{\mu}{\rightarrow} K_{\bullet}\left(C^{*}(\Gamma)\right) \rightarrow K_{\bullet}\left(C^{*}\left(\Gamma^{a b}\right)\right)=K^{\bullet}\left(J^{\text {orb }}(\Sigma)\right)
$$


and similar maps for the symmetric products

$$
\begin{aligned}
K_{\bullet}^{\Gamma^{n} \rtimes S_{n}}\left(\mathbb{H}^{n}\right) \simeq K_{\bullet}^{\text {orb }}\left(\operatorname{Sym}^{n}(\Sigma)\right) & \stackrel{\mu}{\rightarrow} K_{\bullet}\left(C^{*}\left(\Gamma^{n} \rtimes S_{n}\right)\right) \\
& \rightarrow K_{\bullet}\left(C^{*}\left(\Gamma^{a b}\right)\right)=K^{\bullet}\left(J^{\text {orb }}(\Sigma)\right),
\end{aligned}
$$

where the last map is induced by the quotient map

$$
\Gamma^{n} \rtimes S_{n} \rightarrow \Gamma^{n} \rtimes S_{n} /\left\langle S_{n}\right\rangle \simeq \Gamma^{a b} .
$$

\section{The magnetic field and twisted group algebras}

\subsection{Twisted group ring and twisted group $C^{*}$-algebra}

Recall that, for a discrete group $\Gamma$, a multiplier on $\Gamma$ is defined as a map $\sigma: \Gamma \times \Gamma \rightarrow U(1)$ satisfying the properties:

1) $\sigma(\gamma, 1)=\sigma(1, \gamma)=1$, for all $\gamma \in \Gamma$,

2) $\sigma\left(\gamma_{1}, \gamma_{2}\right) \sigma\left(\gamma_{1} \gamma_{2}, \gamma_{3}\right)=\sigma\left(\gamma_{1}, \gamma_{2} \gamma_{3}\right) \sigma\left(\gamma_{2}, \gamma_{3}\right)$, for all $\gamma_{1}, \gamma_{2}, \gamma_{3} \in \Gamma$.

The reduced twisted group $C^{*}$-algebra $C_{r}^{*}(\Gamma, \sigma)$ is the norm closure in the algebra of bounded operators on $\ell^{2}(\Gamma)$ of the twisted group ring $\mathbb{C}[\Gamma, \sigma]$, generated by the left translations $L_{\gamma}^{\sigma}$ with relations $L_{\gamma}^{\sigma} L_{\gamma^{\prime}}^{\sigma}=\sigma\left(\gamma, \gamma^{\prime}\right) L_{\gamma \gamma^{\prime}}^{\sigma}$, represented on $\ell^{2}(\Gamma)$ by the left regular representation

$$
L_{\gamma}^{\sigma} \xi\left(\gamma^{\prime}\right)=\sigma\left(\gamma, \gamma^{-1} \gamma^{\prime}\right) \xi\left(\gamma^{-1} \gamma^{\prime}\right)
$$

\subsection{Cocycles from the magnetic field}

In the system we are considering, we have $n$ indistinguishable particles moving in a negatively curved geometry $\mathbb{H}$, subject to a potential, which is generated by charges disposed along the vertices of an embedding (Cayley graph) of a Fuchsian group $\Gamma$ in $\mathbb{H}$, and to an external magnetic field.

Usually, in the "independent electron approximation" in the theory of solids, one replaces the (unbounded) interaction potential of the manyparticle problem with a Hamiltonian with an effective potential of the form $H=\sum_{i=1}^{n}-\Delta_{x_{i}}+V\left(x_{i}\right)$, with a (bounded) effective potential $V(x)$ of a single-particle problem that encodes an average of the interactions of one of the charge carriers with the others, as well as with the atoms of the periodic medium. Here we consider a more general situation, where the independent electron approximation is perturbed by a (bounded) periodic interaction 
potential. This means that we consider a Hamiltonian as above with the $V\left(x_{i}\right)$ replaced by a smooth bounded function $W\left(x_{1}, \ldots, x_{n}\right)$ on $\mathbb{H}^{n}$, which is invariant under the symmetry group $\Gamma^{n} \rtimes S_{n}$.

As in [14], [49], [50], [51] the magnetic field is described by a closed 2form $\omega=d \eta$ on $\mathbb{H}$. The form is invariant under the action of $\Gamma$ on $\mathbb{H}$, so that $\omega-\gamma^{*} \omega=0$ for all $\gamma \in \Gamma$. However, the potential $\eta$ is not $\Gamma$-invariant. The fact that $d\left(\eta-\gamma^{*} \eta\right)=0$ for all $\gamma \in \Gamma$ implies the existence of a (real valued) 0 -form $\phi_{\gamma}$ on $\mathbb{H}$ satisfying $\gamma^{*} \eta-\eta=d \phi_{\gamma}$, for all $\gamma \in \Gamma$. The function

$$
\phi_{\gamma}=\int_{x_{0}}^{x} \gamma^{*} \eta-\eta
$$

satisfies the property that

$$
\phi_{\gamma}(x)+\phi_{\gamma^{\prime}}(\gamma x)-\phi_{\gamma^{\prime} \gamma}(x)=\phi_{\gamma^{\prime}}\left(\gamma x_{0}\right)
$$

is independent of $x \in \mathbb{H}$. Setting

$$
\sigma\left(\gamma, \gamma^{\prime}\right)=\exp \left(-i \phi_{\gamma^{\prime}}\left(\gamma x_{0}\right)\right)
$$

for a chosen base point $x_{0} \in \mathbb{H}$, determines a multiplier of $\Gamma$, as in $\S 4.1$. The magnetic Laplacian $\Delta^{\eta}=(d-i \eta)^{*}(d-i \eta)$ is invariant under the magnetic translations $T_{\gamma}^{\phi}=e^{-i \phi_{\gamma}} T_{\gamma}$ and the algebra of magnetic translations satisfies

$$
T_{\gamma}^{\phi} T_{\gamma^{\prime}}^{\phi}=\sigma\left(\gamma, \gamma^{\prime}\right) T_{\gamma \gamma^{\prime}}^{\phi}
$$

with $\sigma\left(\gamma, \gamma^{\prime}\right)$ as in (4.4). This follows from (4.3).

Consider now the product $\mathbb{H}^{n}$ and the 2 -form $\varpi=\sum_{j} \omega_{j}$, where $\omega_{j}$ is the pullback $\omega_{j}=\pi_{j}^{*} \omega$ of the magnetic field 2-form described above, under the projection of $\mathbb{H}^{n}$ onto the $j$-th factor. In particular $\omega_{j}$ only depends on the $j$-coordinate of $\mathbb{H}^{n}$.

Lemma 4.1. The 2-form $\varpi$ on $\mathbb{H}^{n}$ is invariant under the action of $\Gamma_{n}=$ $\Gamma^{n} \rtimes S_{n}$. The potential, given by the 1 -form $\zeta=\sum_{j} \eta_{j}$ is invariant under $S_{n}$, hence it descends to a 1-form on $\operatorname{Sym}^{n}(\mathbb{H})=\mathbb{H}^{n} / S_{n}$. The form $\zeta$, moreover, satisfies $g^{*} \zeta-\zeta=d \psi_{g}$, for $g=(\gamma, \sigma) \in \Gamma^{n} \rtimes S_{n}$, with

$$
\psi_{g}(x)=\sum_{j=1}^{n} \phi_{\gamma_{j}}\left(x_{\sigma(j)}\right),
$$


with $\phi_{\gamma_{j}}$ as in (4.2) with a base point $x_{(0)}=\left(x_{0, j}\right)$. This function $\psi_{g}: \mathbb{H}^{n} \rightarrow$ $\mathbb{R}$ satisfies

$$
\psi_{g}(x)+\psi_{g^{\prime}}(g x)-\psi_{g^{\prime} g}(x)=\psi_{g^{\prime}}\left(g x_{(0)}\right)
$$

independent of $x=\left(x_{j}\right) \in \mathbb{H}^{n}$. This determines a multiplier $\sigma_{n}: \Gamma_{n} \times \Gamma_{n} \rightarrow$ $U(1)$

$$
\sigma_{n}\left(g, g^{\prime}\right)=\exp \left(-i \phi_{g^{\prime}}\left(g x_{(0)}\right)\right)
$$

For $g \in \Gamma_{n}$, the magnetic translations $T_{g}^{\psi}$ on $\mathbb{H}^{n}$ satisfy

$$
T_{g^{\prime}}^{\psi} T_{g}^{\psi}=\sigma_{n}\left(g, g^{\prime}\right) T_{g^{\prime} g}^{\psi}
$$

Proof. The identity (4.3) implies that for all $j=1, \ldots, n$ and all $\gamma_{j} \in \Gamma$, and all $\sigma, \sigma^{\prime} \in S_{n}$ we have

$$
\phi_{\gamma_{\sigma^{\prime}(j)}}\left(x_{\sigma^{\prime} \sigma(j)}\right)+\phi_{\gamma_{j}^{\prime}}\left(\gamma_{\sigma^{\prime}(j)} x_{\sigma^{\prime} \sigma(j)}\right)-\phi_{\gamma_{j}^{\prime} \gamma_{\sigma^{\prime}(j)}}\left(x_{\sigma^{\prime} \sigma(j)}\right)=\phi_{\gamma_{j}^{\prime}}\left(\gamma_{\sigma^{\prime}(j)} x_{0, \sigma^{\prime} \sigma(j)}\right),
$$

so that by summing over $j$ we obtain (4.7). The composition of two magnetic translations then gives

$$
e^{-i \psi_{g}(x)} e^{-i \psi_{g^{\prime}}(g x)} f\left(g^{\prime} g x\right)=e^{i\left(\psi_{g}(x)+\psi_{g^{\prime}}(g x)-\psi_{g^{\prime} g}(x)\right)} T_{g^{\prime} g}^{\psi} f(x),
$$

so we obtain (4.9) with the multiplier (4.8).

\section{3. $K$-theory of the twisted group algebra}

The multiplier $\sigma_{n}: \Gamma_{n} \times \Gamma_{n} \rightarrow U(1)$ described above determines a twisting $C_{r}^{*}\left(\Gamma_{n}, \sigma_{n}\right)$ of the group $C^{*}$-algebra. The twisted group algebra provides the algebra of observables for the $n$-particle system, in the presence of the external magnetic field.

In [49] it was shown that the $K$-theory of the twisted group algebra $C_{r}^{*}(\Gamma, \sigma)$ is isomorphic to the $K$-theory of the untwisted algebra, whenever the multiplier $\sigma: \Gamma \times \Gamma \rightarrow U(1)$ has trivial Dixmier-Douady class $\delta(\sigma)=0$. In the case of the algebras $C_{r}^{*}\left(\Gamma_{n}, \sigma_{n}\right)$, we have a similar results, as we will now discuss.

Proposition 4.2. Let $\sigma: \Gamma \times \Gamma \rightarrow U(1)$ be a multiplier, with trivial Dixmier-Douady class, $\delta[\sigma]=0$. Then it determines a multiplier $\sigma_{n}: \Gamma_{n} \times$ $\Gamma_{n} \rightarrow U(1)$, which also has trivial Dixmier-Douady invariants, $\delta\left[\sigma_{n}\right]=0$. 
Proof. Recall that the exponential sequence

$$
1 \rightarrow \mathbb{Z} \stackrel{\iota}{\rightarrow} \mathbb{R} \stackrel{e}{\rightarrow} U(1) \rightarrow 1
$$

with $e(t)=\exp (2 \pi i t)$, determines a long exact cohomology sequence

$$
\begin{aligned}
& \cdots \rightarrow H^{2}(\Gamma, \mathbb{Z}) \stackrel{\iota_{*}}{\longrightarrow} H^{2}(\Gamma, \mathbb{R}) \stackrel{e_{*}}{\longrightarrow} H^{2}(\Gamma, U(1)) \\
& \stackrel{\delta}{\longrightarrow} H^{3}(\Gamma, \mathbb{Z}) \stackrel{\iota_{*}}{\longrightarrow} H^{3}(\Gamma, \mathbb{R}) \rightarrow \cdots
\end{aligned}
$$

where $\delta: H^{2}(\Gamma, U(1)) \rightarrow H^{3}(\Gamma, \mathbb{Z})$ is the Dixmier-Douady map. The Fuchsian group $\Gamma=\Gamma(g, \underline{\nu})$ has cohomology (see [49])

$$
H^{j}(\Gamma, \mathbb{R})= \begin{cases}\mathbb{R} & j=0,2 \\ \mathbb{R}^{2 g} & j=1 \\ 0 & j \geq 3\end{cases}
$$

A multiplier $\sigma: \Gamma \times \Gamma \rightarrow U(1)$ determines a cocycle $\sigma \in Z^{2}(\Gamma, U(1))$, with cohomology class $[\sigma] \in H^{2}(\Gamma, U(1))$. If the Dixmier-Douady class $\delta[\sigma]=0$ in $H^{3}(\Gamma, \mathbb{Z})$, then the class $[\sigma]$ is in the range of the map $e_{*}: H^{2}(\Gamma, \mathbb{R}) \rightarrow$ $H^{2}(\Gamma, U(1))$, that is, there exists a cocycle $\xi \in Z^{2}(\Gamma, \mathbb{R})$ such that $[\sigma]=$ $[e(\xi)]$. Using the branched covering of the 2-dimensional orbifold $\Sigma$ by a smooth Riemann surface $\Sigma_{g^{\prime}}$ with $\Sigma=\Sigma_{g^{\prime}} / G$, for a finite group $G$, we can identify $H^{2}(\Gamma, \mathbb{R}) \cong H^{2}\left(\Sigma_{g^{\prime}}, \mathbb{R}\right)$, since the finite group $G$ has no nontrival cohomology with real coefficients. Thus, we can realize the cocyle $\xi$ in terms of a closed 2 -form $\omega$ on $\Sigma_{g^{\prime}}$, or of its $\Gamma_{g^{\prime}}$-invariant lift to the universal cover $\mathbb{H}$, with $[\sigma]=[e(\omega)]$. Consider then the cohomology $H^{2}\left(\Gamma_{n}, \mathbb{R}\right)$. By the results of [47] [55] we know that the Lyndon-Hochschild-Serre spectral sequence for the group cohomology of the wreath product $\Gamma^{n} \rtimes S_{n}$ degenerates at the $E_{2}$-term, for both integral cohomology and cohomology with coefficients in a field. In particular, this means that we can compute the cohomology of $\Gamma_{n}$ with real coefficients in terms of the cohomology groups $E_{2}^{p q}=H^{p}\left(S_{n}, H^{q}\left(\Gamma^{n}, \mathbb{R}\right)\right)$, with $H^{q}\left(\Gamma^{n}, \mathbb{R}\right)=\oplus_{i_{1}+\cdots+i_{n}=q} H^{i_{1}}(\Gamma, \mathbb{R}) \times$ $\cdots \times H^{i_{n}}(\Gamma, \mathbb{R})$. For $p+q=2$, the only non-trivial term is $H^{0}\left(S_{n}, H^{2}\left(\Gamma^{n}, \mathbb{R}\right)\right)$, since for the symmetric group $H^{j}\left(S_{n}, \mathbb{R}\right)=0$ for $j \geq 1$. There is a subspace in $H^{0}\left(S_{n}, H^{2}\left(\Gamma^{n}, \mathbb{R}\right)\right) \cong H^{2}\left(\Gamma^{n}, \mathbb{R}\right)$ that is isomorphic to $H^{2}(\Gamma, \mathbb{R})^{\oplus n}$, namely the subspace given by the Künneth components involving only $H^{2}$ and $H^{0}$ and not $H^{1}$. This subspace can be identified with $H^{2}\left(\Sigma_{g^{\prime}}, \mathbb{R}\right)^{\oplus n}$, as above. Using this identification, we see that the closed 2-form $\varpi(x)=\sum_{j} \omega\left(x_{j}\right)$ on $\Sigma_{g^{\prime}}^{n}$ determines a class $[\varpi]$ in this subspace of $H^{2}\left(\Gamma_{n}, \mathbb{R}\right)$, hence it determines 
a multiplier class $\left[\sigma_{n}\right]=[e(\varpi)] \in H^{2}\left(\Gamma_{n}, U(1)\right)$, as the image under the map $e_{*}$ in the cohomology exact sequence

$$
\begin{aligned}
\cdots \rightarrow H^{2}\left(\Gamma_{n}, \mathbb{Z}\right) \stackrel{\stackrel{\iota_{*}}{\longrightarrow}}{\longrightarrow} H^{2}\left(\Gamma_{n}, \mathbb{R}\right) \stackrel{e_{*}}{\longrightarrow} H^{2}\left(\Gamma_{n}, U(1)\right) \\
\stackrel{\delta}{\longrightarrow} H^{3}\left(\Gamma_{n}, \mathbb{Z}\right) \stackrel{\iota_{*}}{\longrightarrow} H^{3}\left(\Gamma_{n}, \mathbb{R}\right) \rightarrow \cdots
\end{aligned}
$$

By construction, a representative $\sigma_{n}: \Gamma_{n} \times \Gamma_{n} \rightarrow U(1)$ of this class will be a multiplier with trivial Dixmier-Douady class, $\delta\left[\sigma_{n}\right]=0$.

Remark 4.3. In the case of the multiplier $\sigma: \Gamma \times \Gamma \rightarrow U(1)$ defined by the magnetic field, we can take, in the argument of Lemma 4.2, the $\Gamma$ invariant 2-form $\omega$ on $\mathbb{H}$ given by the magnetic field. The corresponding multiplier $\sigma_{n}: \Gamma_{n} \times \Gamma_{n} \rightarrow U(1)$ will then agree with the one constructed in the previous subsection. Thus, the $\sigma$ and the $\sigma_{n}$ determined by the magnetic field have trivial Dixmier-Douady invariant.

With the $K$-amenability property discussed in $\S 4.4$ below, we have the following.

Proposition 4.4. Let $\sigma_{n}: \Gamma_{n} \times \Gamma_{n} \rightarrow U(1)$ be a multiplier as above, with $\delta\left[\sigma_{n}\right]=0$. Then $K_{\bullet}\left(C_{r}^{*}\left(\Gamma_{n}, \sigma_{n}\right)\right) \simeq K_{\bullet}\left(C_{r}^{*}\left(\Gamma_{n}\right)\right)$.

Proof. The argument is the same as in [49]. If $\delta\left[\sigma_{n}\right]=0$, we have $\left[\sigma_{n}\right]=$ $\left[e\left(\xi_{n}\right)\right]$ for some $\xi_{n} \in Z^{2}\left(\Gamma_{n}, \mathbb{R}\right)$, by (4.11). We can then use a homotopy $\left[\sigma_{n, t}\right]=\left[e\left(t \xi_{n}\right)\right]$ with $0 \leq t \leq 1$. Consider the discrete subgroup $\Gamma_{n}$ of $\mathcal{G}_{n}=$ $\operatorname{PSL}(2, \mathbb{R})^{n} \rtimes S_{n}$, with quotient $\Gamma_{n} \backslash \mathcal{G}_{n}=\mathcal{P}_{n}$ and let $\mathcal{A}$ be an algebra with an action of $\Gamma_{n}$ by automorphisms. The crossed product $\left(\mathcal{A} \otimes C_{0}\left(\mathcal{G}_{n}\right)\right) \rtimes \Gamma_{n}$ is Morita equivalent to the algebra of sections $C_{0}\left(\Gamma_{n} \backslash \mathcal{G}_{n}, \mathcal{E}\right)$ of the flat $\mathcal{A}$ bundle $\mathcal{E} \rightarrow \hat{\mathcal{P}}_{n}$ with $\mathcal{E}=\left(\mathcal{A} \times \mathcal{G}_{n}\right) / \Gamma_{n}$ with the quotient taken with respect to the diagonal action. Moreover, the algebras $\left(\mathcal{A} \rtimes \Gamma_{n}\right) \otimes C_{0}\left(\mathcal{G}_{n}\right)$ and $(\mathcal{A} \otimes$ $\left.C_{0}\left(\mathcal{G}_{n}\right)\right) \rtimes \Gamma_{n}$ have the same $\mathcal{K}_{n}$-equivariant $K$-theory. Combined with the previous Morita equivalence and the fact that $\mathcal{G}_{n} / \mathcal{K}_{n}=\mathbb{H}^{n}$, we obtain

$$
K_{\mathcal{K}_{n}, \bullet}\left(C_{0}\left(\mathcal{P}_{n}, \mathcal{E}\right)\right) \cong K_{\mathcal{K}_{n}, \bullet+\operatorname{dim}\left(\mathcal{G}_{n} / \mathcal{K}_{n}\right)}\left(\mathcal{A} \rtimes \Gamma_{n}\right)=K_{\mathcal{K}_{n}, \bullet}\left(\mathcal{A} \rtimes \Gamma_{n}\right)
$$

As in [49], we use the Packer-Raeburn stabilization trick [59]. The algebra $\mathcal{A} \rtimes_{\sigma_{n}} \Gamma_{n}$ is stably isomorphic to $(\mathcal{A} \otimes \mathbb{K}) \rtimes \Gamma$ with $\mathbb{K}$ the algebra of compact 
operators, and we consider the flat $\mathcal{A} \otimes \mathbb{K}$-bundle

$$
\mathcal{E}_{\sigma_{n}}=\left(\mathcal{A} \otimes \mathbb{K} \times \mathcal{G}_{n}\right) / \Gamma_{n} \rightarrow \Gamma_{n} \backslash \mathcal{G}_{n} .
$$

As in Proposition 2.2 of [49], we then have

$$
K_{\bullet}\left(C^{*}\left(\Gamma_{n}, \sigma_{n}\right)\right) \cong K_{\mathcal{K}}^{\bullet}\left(\Gamma_{n} \backslash \mathcal{G}_{n}, \delta\left(B_{\sigma_{n}}\right)\right) .
$$

Here the twisted $\mathcal{K}_{n}$-equivariant $K$-theory $K_{\mathcal{K}}^{\bullet}\left(\Gamma_{n} \backslash \mathcal{G}_{n}, \delta\left(B_{\sigma_{n}}\right)\right)$ is the same as the $\mathcal{K}_{n}$-equivariant $K$-theory of the continuous trace $C^{*}$-algebra $B_{\sigma_{n}}=$ $C_{0}\left(\Gamma_{n} \backslash \mathcal{G}_{n}, \mathcal{E}_{\sigma_{n}}\right)$ with Dixmier-Douady class $\delta\left(B_{\sigma_{n}}\right)$. By Theorem 2.3 of [49], using the $K$-amenability property of $\S 4.4$ below, we then have $K_{\bullet}\left(C^{*}\left(\Gamma_{n}, \sigma_{n}\right)\right)$ $\cong K_{\bullet}\left(C_{r}^{*}\left(\Gamma_{n}, \sigma_{n}\right)\right)$. We then obtain isomorphisms $K_{\bullet}\left(C_{r}^{*}\left(\Gamma_{n}, \sigma_{n, t}\right)\right)=$ $K_{\bullet}\left(C_{r}^{*}\left(\Gamma_{n}\right)\right)$.

Combining the isomorphism $K_{\bullet}\left(C_{r}^{*}\left(\Gamma_{n}, \sigma_{n, t}\right)\right)=K_{\bullet}\left(C_{r}^{*}\left(\Gamma_{n}\right)\right)$ obtained above with the Kasparov assembly map, we obtain a twisted Kasparov map (as in [49], [50])

$$
\mu_{\sigma_{n}}: K_{\text {orb }}^{\bullet}\left(\operatorname{Sym}^{n}(\Sigma)\right) \rightarrow K_{\bullet}\left(C_{r}^{*}\left(\Gamma_{n}, \sigma_{n}\right)\right) .
$$

\subsection{K-amenability}

We now turn to the K-amenability property of the group $\mathrm{SL}(2, \mathbb{R})^{n} \rtimes S_{n}$. We first recall some basic facts about K-amenability. A locally compact second countable group $\mathcal{G}$ is amenable if the map $\lambda: C^{*}(\mathcal{G}) \rightarrow C_{r}^{*}(\mathcal{G})$ determined by restriction of representations is an isomorphism. In particular, if $\mathcal{G}$ acts on a $C^{*}$-algebra $A$ as a $C^{*}$-dynamical system, and $\mathcal{G}$ is amenable, then the map $\lambda_{A}: C^{*}(G, A) \rightarrow C_{r}^{*}(G, A)$ is also an isomorphism. The notion of $\mathrm{K}$-amenability expresses a weaker $\mathrm{K}$-theoretic form of this property. We consider here two forms of the K-amenability property, as in [30]. Recall that a Fredholm $\mathcal{G}$-module is a pair $(\mathcal{H}, F)$ of a Hilbert space $\mathcal{H}=\mathcal{H}_{0} \oplus \mathcal{H}_{1}$ with unitary representations $\rho_{0}, \rho_{1}$ of $\mathcal{G}$ on $\mathcal{H}_{0}$ and $\mathcal{H}_{1}$ and with a bounded operator $F: \mathcal{H}_{0} \rightarrow \mathcal{H}_{1}$ with

$$
g \mapsto \rho_{1}(g) \circ F-F \circ \rho_{0}(g)
$$

a compact operator and with $F^{*} F-1$ and $F F^{*}-1$ also compact operators. The trivial Fredholm module has $\mathcal{H}_{0}=\mathbb{C}$ and $\mathcal{H}_{1}=0$. The notion of a homotopy of Fredholm module is similarly stated (see e.g. [30]). 
1) $\mathcal{G}$ is K-amenable if, for any $C^{*}$-dynamical system $(\mathcal{G}, A)$, the map $\lambda_{A *}$ : $K_{*}\left(C^{*}(G, A)\right) \rightarrow K_{*}\left(C_{r}^{*}(G, A)\right)$ is an isomorphism.

2) $\mathcal{G}$ is $\mathrm{K}$-amenable if there exists a Fredholm $\mathcal{G}$-module $(\mathcal{H}, F)$ such that the representation of $\mathcal{G}$ on the Hilbert space $\mathcal{H}$ is weakly contained in the left regular representation, with $(\mathcal{H}, F)$ homotopic to the trivial Fredholm $\mathcal{G}$-module.

The second version of the K-amenability property implies the first: we are going to refer to this second property as K-amenability. It was shown in [43] that any covering group of the identity component of $S O(n, 1)$ is $\mathrm{K}$-amenable. In particular, $\mathrm{SL}(2, \mathbb{R})$ is $\mathrm{K}$-amenable. It is also shown in [19] that the class of $\mathrm{K}$-amenable groups is closed with respect to the operations of taking subgroups and taking direct products. Thus, the groups $\operatorname{SL}(2, \mathbb{R})^{n}$ are K-amenable.

Proposition 4.5. The wreath product groups $\mathrm{SL}(2, \mathbb{R})^{n} \rtimes S_{n}$ are $K$ amenable.

Proof. The K-amenability of SL $(2, \mathbb{R})$ follows from the general result of [43] mentioned above. A more explicit proof was given in [30], by constructing a Fredholm module with the desired properties. This has $\mathcal{H}_{0}=L^{2}(K / M)$ where $K$ is the maximal compact (the circle group), with basis $\phi_{n}(\theta)=e^{i n \theta}$, $n \in 2 \mathbb{Z}$. and $M=\{ \pm 1\} \subset K$, and $\mathcal{H}_{1}=\mathcal{H}_{+2} \oplus \mathcal{H}_{-2}$ consisting of Hilbert space completions of the two discrete series representations, respectively given by the spans of $\left\{w_{n} \mid n \in 2 \mathbb{Z}, n \geq 2\right\}$ and $\left\{w_{n} \mid n \in 2 \mathbb{Z}, n \leq-2\right\}$, and with $F\left(\phi_{0}\right)=0$ and $F\left(\phi_{n}\right)=w_{n}$. See $\S 1$ of [30] for more details. The Kamenability of $\operatorname{SL}(2, \mathbb{R})^{n}$ follows, as mentioned above, from the general result of [19] which in particular shows the property is preserved by direct products. The construction of a Fredholm module for $\operatorname{SL}(2, \mathbb{R})^{n}$ can be obtained from the construction of [30] by tensor products. Notice that the weak containment of representations has the property that, if unitary representations $\pi_{1}$ and $\pi_{2}$ are, respectively, weakly contained in unitary representations $\rho_{1}$ and $\rho_{2}$, then $\pi_{1} \otimes \pi_{2}$ is weakly contained in $\rho_{1} \otimes \rho_{2}$. Let $\pi$ be a unitary representation of a locally compact group $\mathcal{G}$ on a Hilbert space $\mathcal{H}$. It induces a representation $\mathcal{R}_{\pi}$ of the Banach algebra $L^{1}(\mathcal{G})$ on the same Hilbert space. Moreover, one has a $\star$-homomorphism $\mathcal{R}: L^{1}(\mathcal{G}) \rightarrow C_{r}^{*}(\mathcal{G})$. The representation $\pi$ is weakly contained in the regular representation if $\left\|\mathcal{R}_{\pi}(f)\right\| \leq\|\mathcal{R}(f)\|$ for all $f \in L^{1}(\mathcal{G})$, see Definition 9.2.7 of [32]. For $\mathcal{G}_{n}=\operatorname{SL}(2, \mathbb{R})^{n} \rtimes S_{n}$, consider the $\mathcal{G}_{n}$-Fredholm module given by $\mathcal{H}^{\otimes n}$, with $\mathcal{H}$ the Fredholm module of [30]. The operator induced by $F$ commutes with elements of $S_{n}$, and one 
obtains in this way a $\mathcal{G}_{n}$-Fredholm module. The representation of $\mathcal{G}_{n}$ is still weakly contained in the regular representation, because the inequality above is still satisfied for $f \in L^{1}\left(\mathcal{G}_{n}\right)$.

As an alternative, it may also be possible prove the result above by adapting the argument in Proposition 2.5 and Corollary 2.6 of [19].

\section{Different notions of orbifold Euler characteristic}

There are several different notions of orbifold Euler characteristic used in the literature. We will recall here some of the main versions and their relation. One of the main difference is that some orbifold Euler characteristics are rational valued, while other, even though they appear to be defined as fractions, are in fact integer valued. In particular, we are interested here in distinguishing between the Satake notion of (rational valued) orbifold Euler characteristic, which plays an important role in the noncommutative geometry approach to the fractional quantum Hall effect developed in [49], [50], [51], and the notion of orbifold Euler characteristic that arises naturally in string theory, [21], [69]. It was shown in [37], by a simple calculation, that the latter is integer valued. We restrict our attention here to the case of good orbifolds, which are global quotients, since the specific cases we intend to focus on, the symmetric products $\operatorname{Sym}^{n}(\Sigma)$ of good 2-dimensional orbifolds, belong to this class: they are global quotients $\operatorname{Sym}^{n}(\Sigma)=\Sigma_{g^{\prime}}^{n} / G^{n} \rtimes S_{n}$, as we discussed above.

Let $X$ be a smooth manifold and $G$ a finite group, acting on $X$ with an orbifold quotient $X / G$. Then the Satake orbifold Euler characteristic, [60], which we simply write as $\chi^{\text {orb }}(X / G)$ is given by

$$
\chi^{o r b}(X / G)=\frac{1}{\# G} \chi(X) \in \mathbb{Q}
$$

In [60] a Gauss-Bonnet theorem is proved for orbifolds, where the usual topological Euler characteristic is replaced by the orbifold version (5.1), which is no longer, in general, an integer. The index theorems for elliptic operators on orbifolds proved in [44], [45] generalize the result of [60]. They were used in [49], [50] to obtain fractional values of the Hall conductance as values of a higher twisted index theorem modeled on [44] and on the higher index theorem of [17].

A different notion of orbifold Euler characteristic arises naturally in the context of string theory on orbifolds, [21], [69]. We will refer to it here as "string-theoretic orbifold Euler characteristic", and we will denote it by 
$\chi^{\text {orb }}(X, G)$, again assuming that the orbifold is a global quotient $X / G$ of a smooth manifold by a finite group action. This version of the orbifold Euler characteristic is defined as

$$
\chi^{\text {orb }}(X, G)=\frac{1}{\# G} \sum_{g h=h g} \chi\left(X^{\langle g, h\rangle}\right),
$$

where the sum is over all pairs of commuting elements in $G$ and $X^{\langle g, h\rangle}$ is the (common) fixed point set of $g$ and $h$. Although from this definition this also appears to be rational valued, it is shown in [37] that the sum in (5.2) can be equivalently written as

$$
\chi^{o r b}(X, G)=\sum_{[g]} \chi\left(X^{g} / C(g)\right),
$$

where now the sum is over conjugacy classes $[g]$ and $C(g)$ is the centralizer of $g$ in $G$. The denominator $\# G$ disappears due to the simple fact that $\#[g] \cdot \# C(g)=\# G$. In orbifold string theory, the sum in (5.2) corresponds to the sum over the different sectors. Notice that the Satake orbifold Euler characteristic $\chi^{\text {orb }}(X / G)$ appears in the sum (5.2) as the term corresponding to the trivial sector with $g=h=1$. It was shown in [4] that $\chi^{\text {orb }}(X, G)=\operatorname{rank} K_{G}^{0}(X)-\operatorname{rank} K_{G}^{1}(X)$, the difference of ranks of the equivariant $K$-theory.

Example 5.1. In the case of the 2-dimensional good orbifold $\Sigma=\Sigma_{g^{\prime}} / G$ the Satake orbifold Euler characteristic is given by $\chi^{\text {orb }}(\Sigma)=(\# G)^{-1} \chi\left(\Sigma_{g^{\prime}}\right)$ $=\chi(\Sigma)+\sum_{j}\left(\nu_{j}^{-1}-1\right)$, while the string-theoretic orbifold Euler characteristic is $\chi^{\text {orb }}\left(\Sigma_{g^{\prime}}, G\right)=\chi\left(\Sigma_{g^{\prime}}\right)+\sum_{j}\left(\nu_{j}-1\right)$.

\subsection{Orbifolds of $A$-sectors and inertia orbifolds}

The Satake orbifold Euler characteristic and the string-theoretic orbifold Euler characteristic admit a family of common generalizations, see [66], [67] and in [27], [28], [29]. As above, let $Y=X / G$ be a good orbifold. We denote by $\mathcal{G}=\mathcal{G}(Y)$ the associated orbifold groupoid. In this setting one considers the additional data of a finitely generated discrete group $A$ and defines the orbifold $Y_{A}$ of $A$-sectors of $Y$ through its orbifold groupoid, which is given by $\mathcal{G}\left(Y_{A}\right)=\mathcal{G}(Y) \ltimes \operatorname{Hom}(A, \mathcal{G}(Y))$. One then defines the orbifold Euler characteristic $\chi_{A}^{o r b}(X, G)$ as

$$
\chi_{A}^{\text {orb }}(X, G)=\chi^{\text {orb }}\left(Y_{A}\right),
$$


namely the Satake orbifold Euler characteristic of the orbifold $Y_{A}$. When $A=\mathbb{Z}^{m}$ one recovers the orbifold Euler characteristics $\chi_{m}^{\text {orb }}(X, G)$ of [13]. In particular, $\chi_{\mathbb{Z}^{2}}^{\text {orb }}(X, G)=\chi^{\text {orb }}(X, G)$, with $Y_{\mathbb{Z}^{2}}$ the inertia orbifold.

\subsection{Orbifold Chern-Schwartz-MacPherson classes}

A generalization of the generating function of (string theoretic) orbifold Euler characteristics (5.10) was given in [66], by considering the orbifold Euler characteristics $\chi_{A}^{\text {orb }}\left(X^{n}, G^{n} \rtimes S_{n}\right)$, with $G$ a finite group. The case of the string-theoretic orbifold Euler characteristics of (5.2) and (5.10) is recovered for $A=\mathbb{Z}^{2}$. A further generalization of both (5.10) and the result of [66] was obtained in [56] as a generating function of orbifold characteristic classes, where the latter are defined as (equivariant) Chern-Schwatz-MacPherson classes, whose zero-dimensional component recovers the Euler characteristic. The notion of orbifold Chern-Schwatz-MacPherson classes considered in [56] is closely related to the stringy Chern classes of [2] and [20]. The orbifold CSM class is defined in [56] as the image under the equivariant MacPherson natural transformation $C_{*}^{G_{n}}$ (see [57]) of the canonical constructible function

$$
\mathbf{1}_{\Sigma_{g^{\prime}}^{n}, G_{n}}^{\mathbb{Z}^{2}}=\frac{1}{\# G_{n}} \sum_{\rho \in \operatorname{Hom}\left(\mathbb{Z}^{2}, G_{n}\right)} \mathbf{1}_{\left(\Sigma_{g^{\prime}}^{n}\right) \rho\left(\mathbb{Z}^{2}\right)}
$$

where $\left(\Sigma_{g^{\prime}}^{n}\right)^{\rho\left(\mathbb{Z}^{2}\right)}$ is the fixed point set of the action of $\rho\left(\mathbb{Z}^{2}\right) \subset G_{n}$ on $\Sigma_{g^{\prime}}^{n}$. Then the generating function of the orbifold CSM classes is then obtained by applying $C_{*}^{G, s y m}=\prod_{n} C_{*}^{G_{n}}$ to the series $\sum_{n} \mathbf{1}_{\Sigma_{g^{\prime}}^{n}, G_{n}}^{\mathbb{Z}^{2}} q^{n}$. This gives (Proposition 4.2 of [56])

$$
\sum_{n} C_{*}^{G_{n}}\left(\Sigma_{g^{\prime}}^{n}\right) q^{n}=\prod_{\ell=1}^{\infty}\left(1-q^{\ell} \Delta^{\ell}\right)^{-C_{*}^{G}\left(\Sigma_{g^{\prime}}\right)}
$$

as in Theorem 1.2 of [56] with $A=\mathbb{Z}^{2}$, and with $\Delta^{\ell}$ the morphism on homology induced by the diagonal embedding $\Delta: \Sigma_{g^{\prime}} \hookrightarrow \Sigma_{g^{\prime}}^{\ell}$. The string-theoretic orbifold Euler characteristics (5.10) are obtained by taking the 0-component of the CSM class, see (2.2) of [56]. Moreover, one can view the orbifold CSM class as in (2.2) and (2.4) of [56], as a sum

$$
C_{*}^{o r b}(X / G)=C_{*}\left(\pi_{*} \mathbf{1}_{X, G}^{\mathbb{Z}^{2}}\right)=\pi_{*} \iota^{*} C_{*}^{G}\left(\mathbf{1}_{X, G}^{\mathbb{Z}^{2}}\right)=\sum_{g} \iota_{g}^{*} C_{*}\left(X^{g} / C(g)\right),
$$


where $\pi$ maps $G$-invariant constructible functions on $X$ to constructible functions on $X / G$ and $\iota^{*}$ is the homomorphism from $H_{*}^{G}(X)$ to $H_{*}(X)$ (inclusion of $X$ as fiber of $X \times_{G} E G \rightarrow B G$ ); the last sum is over conjugacy classes of elements $g \in G$, and $C(g)$ is the centralizer of $g$. In the latter form, these classes can be viewed (after reinterpreting them cohomologically) as residing in the delocalized equivariant cohomology $H^{*}(X, G)=\oplus H^{*}\left(X^{g}\right)^{C(g)}$, see [7]. Delocalized equivariant cohomology for symmetric products was considered in [72]. In the more general case of orbifolds $X^{n} / G_{n}$ with a wreath product $G_{n}=G^{n} \rtimes S_{n}$, the delocalized equivariant cohomology is obtained as image under the Connes-Chern character of the $K$-theoretic construction of [71] recalled in $§ 5.3$ below. Delocalized equivariant cohomology is also the natural cohomology for string theory on orbifolds, in the sense of [21], [69].

Although the Chern-Schwartz-MacPherson classes are defined as homological Chern classes of singular varieties, they still admit a Chern-Weil type formulation in terms of curvature forms (currents), as shown in [31]. The construction of Chern-Weil representatives is based on an embedding of the singular variety $X$ in a smooth ambient variety $M$, and in universal differential forms (currents) $\gamma_{k}$ obtained from the pullbacks to $\mathbb{P}\left(T^{*} M\right)$ of the Chern classes $C_{k}(M)$ and the powers $\zeta^{r}$ of a 2 -form $\zeta$ on $\mathbb{P}\left(T^{*} M\right)$ determined by the property that, on the total space $\mathbb{S}\left(T^{*} M\right)$ of the Hopf bundle $\pi_{H}: \mathbb{S}\left(T^{*} M\right) \rightarrow \mathbb{P}\left(T^{*} M\right)$ with fiber $S^{1}$, one has $\pi_{H}^{*} \zeta=d \beta$, with $\beta$ the generator of the cohomology of the fiber $S^{1}$. More precisely, one has ( $\S 2$ of [31])

$$
\left(\sum_{r \geq 0} \zeta^{r}\right) \wedge \pi^{*} C_{*}\left(T^{*} M\right)=\sum_{k}(-1)^{\operatorname{dim} M-k} \gamma_{k}
$$

which, using the Chern-Weil curvature forms for $C_{*}\left(T^{*} M\right)$ leads to ChernWeil representatives for the $\gamma_{k}$ The Chern-Schwartz-MacPherson class of $X \subset M$ is then obtained as the current $C_{*}(X)$ whose pairing with a form $\omega$ is given by

$$
\left\langle C_{k}(X), \omega\right\rangle=\left\langle\mathbb{P}\left(N^{*}(X)\right), \gamma_{k} \wedge \omega\right\rangle=\int_{\mathbb{P}\left(N^{*}(X)\right)} \gamma_{k} \wedge \omega
$$

where $N^{*}(X)$ is the Legendrian conormal cycle defined in $\S 1$ of [31] and $\mathbb{P}\left(N^{*}(X)\right)=\pi_{\mathcal{H}, \sharp}\left(N^{*}(X)\lfloor\beta)\right.$, that is, $N^{*}(X)$ is the extension of $\mathbb{P}\left(N^{*}(X)\right)$ to the total space of the Hopf bundle $\mathcal{H}, N^{*}(X)=\mathbb{P}\left(N^{*}(X)\right) \times_{\mathcal{H}}\left[S^{1}\right]$. In case of a smooth variety $X$ one has $N^{*}(X)=(-1)^{\operatorname{dim} M-\operatorname{dim} X}\left[\mathbb{P}\left(\nu^{*}(X)\right)\right]$ where $\nu^{*}(X)$ is the conormal bundle. 


\subsection{The Fock space of orbifold symmetric products}

Following results of Segal for the equivariant $K$-theory of symmetric products [64], Weiqiang Wang established in [71] analogous results for the more general case of wreath products $G \sim S_{n}=G^{n} \rtimes S_{n}$, for a finite group $G$ acting on a locally compact Hausdorff paracompact $G$-space $X$.

We use the notation $G_{n}=G^{n} \rtimes S_{n}$ as in [71]. Let $K_{G_{n}}^{\bullet}\left(X^{n}\right)$ be the equivariant $K$-theory and let $K_{G_{n}, \mathbb{C}}^{\bullet}\left(X^{n}\right)=K_{G_{n}}^{\bullet}\left(X^{n}\right) \otimes_{\mathbb{Z}} \mathbb{C}$. The Fock space $\mathcal{F}_{G}(X)$ is given by

$$
\mathcal{F}_{G}(X):=\oplus_{n \geq 0} q^{n} K_{G^{n} \rtimes S_{n}, \mathbb{C}}\left(X^{n}\right),
$$

with $q$ a formal variable (which keeps count of the graded structure) and with the term $n=0$ equal to $\mathbb{C}$. It is proved in [71] that the Fock space $\mathcal{F}_{G}(X)$ has the following properties:

- $\mathcal{F}_{G}(X)$ is a graded connected Hopf algebra with multiplication defined by

$$
K_{G_{n}, \mathbb{C}}^{\bullet}\left(X^{n}\right) \otimes K_{G_{m}, \mathbb{C}}^{\bullet}\left(X^{m}\right) \stackrel{K}{\rightarrow} K_{G_{n} \times G_{m}, \mathbb{C}}^{\bullet}\left(X^{n+m}\right) \stackrel{\text { Ind }}{\rightarrow} K_{G_{n+m}, \mathbb{C}}^{\bullet}\left(X^{n}\right),
$$

where the first map $K$ is the Künneth isomorphism and the second map Ind is the induction maps for subgroups; the comultiplication is given by

$$
\begin{aligned}
K_{G_{n}, \mathbb{C}}^{\bullet}\left(X^{n}\right) & \stackrel{R}{\rightarrow} \oplus_{m=0}^{n} K_{G_{m} \times G_{n-m}, \mathbb{C}}^{\bullet}\left(X^{n}\right) \\
& \stackrel{K^{-1}}{\rightarrow} \oplus_{m=0}^{n} K_{G_{m}, \mathbb{C}}^{\bullet}\left(X^{m}\right) \otimes K_{G_{n-m}, \mathbb{C}}^{\bullet}\left(X^{n-m}\right),
\end{aligned}
$$

where the first map $R$ is restriction from $G_{n}$ to subgroups $G_{m} \times G_{n-m}$ and the second map $K^{-1}$ is the inverse of the Künneth isomorphism.

- As a graded algebra (graded over $\left.\mathbb{Z}^{+} \times \mathbb{Z} / 2 \mathbb{Z}\right), \mathcal{F}_{G}(X)$ is isomorphic to the algebra

$$
\mathcal{S}\left(\oplus_{n \geq 1} q^{n} K_{G, \mathbb{C}}^{0}(X)\right) \otimes \Lambda\left(\oplus_{n \geq 1} q^{n} K_{G, \mathbb{C}}^{1}(X)\right),
$$

where $\mathcal{S}$ denotes the symmetric algebra and $\Lambda$ the exterior algebra. The graded dimension satisfies

$$
\operatorname{dim}_{q} \mathcal{F}_{G}(X)=\sum_{n \geq 0} q^{n} \operatorname{dim} K_{G_{n}, \mathbb{C}}\left(X^{n}\right)=\frac{\prod_{\ell \geq 1}\left(1+q^{\ell}\right)^{\operatorname{dim} K_{G, \mathbb{C}}^{1}(X)}}{\prod_{\ell \geq 1}\left(1-q^{\ell}\right)^{\operatorname{dim} K_{G, \mathbb{C}}^{0}(X)}}
$$


- The orbifold Euler characteristics (in the string theory sense) of the symmetric products have a generating function

$$
\sum_{n \geq 1} \chi^{\text {orb }}\left(X^{n}, G_{n}\right) q^{n}=\prod_{\ell=1}^{\infty}\left(1-q^{\ell}\right)^{-\chi^{o r b}(X, G)} .
$$

- $\mathcal{F}_{G}(X)$ is a free $\lambda$-ring generated by $K_{G, \mathbb{C}}^{\bullet}(X)$.

Remark 5.2. This construction, applied to $X=\Sigma_{g^{\prime}}$ and $G=\Gamma(g, \underline{\nu}) / \Gamma_{g^{\prime}}$ provides a Fock space $\mathcal{F}_{G}\left(\Sigma_{g^{\prime}}\right)$ for our setting, with all the properties listed above, and

$$
\sum_{n \geq 1} \chi^{o r b}\left(\Sigma_{g^{\prime}}^{n}, G^{n} \rtimes S_{n}\right) q^{n}=\prod_{\ell=1}^{\infty}\left(1-q^{\ell}\right)^{-\chi^{o r b}\left(\Sigma_{g^{\prime}}, G\right)} .
$$

\section{Higher twisted index theory}

Let $\mathcal{E}$ be an orbifold vector bundle on the good 2-dimensional orbifold $\Sigma$. It defines a class $[\mathcal{E}]$ in $K_{\text {orb }}^{\bullet}(\Sigma)$. On the $n$-fold product $\Sigma^{n}$ we consider the orbifold bundle $\mathcal{E}^{\bigotimes n}$. This determines an orbifold vector bundle $\mathcal{E}_{n}$ on the symmetric product $\operatorname{Sym}^{n}(\Sigma)$. Let $\tilde{\mathcal{E}}$ be the pull back of $\mathcal{E}$ to $\mathbb{H}$ and $\tilde{\mathcal{E}}^{\bigotimes n}$ the corresponding bundle on $\mathbb{H}^{n}$. Similarly, we consider the pullback $\mathcal{E}^{\prime}$ to $\Sigma_{g^{\prime}}$ and the bundle $\mathcal{E}^{\prime \otimes n}$ on $\Sigma_{g^{\prime}}^{n}$. The class $\left[\mathcal{E}_{n}\right] \in K_{\text {orb }}^{\bullet}\left(\operatorname{Sym}^{n}(\Sigma)\right)$ corresponds to the classes $\left[\tilde{\mathcal{E}}^{\bigotimes n}\right]$ and $\left[\mathcal{E}^{\prime \otimes n}\right]$, respectively, under the identifications $K_{\text {orb }}^{\bullet}\left(\operatorname{Sym}^{n}(\Sigma)\right)=K_{\Gamma_{n}}^{\bullet}\left(\mathbb{H}^{n}\right)=K_{G_{n}}^{\bullet}\left(\Sigma_{g^{\prime}}^{n}\right)$. Let $\not_{\tilde{\mathcal{E}}}^{+}$be the twisted Dirac operator on $\mathbb{H}$ and let $\nabla=d-i \eta$ be the hermitian connection on $\mathbb{H}$ with curvature $\nabla^{2}=i \omega$, where $\omega$ is the $\Gamma$ invariant 2 -form defined by the magnetic field. The operator $\not_{\tilde{\mathcal{E}}}^{+} \otimes \nabla$ commutes with the projective action $(\Gamma, \sigma)$. Similarly, we write $\not_{\tilde{\mathcal{E}}^{\bigotimes_{n}}}^{+}$for the twisted Dirac operator on $\mathbb{H}^{n}$ and we consider $\not_{\tilde{\mathcal{E}}^{\bigotimes_{n}}}^{+} \otimes \nabla_{n}$ where $\nabla_{n}=d-i \zeta$ where $\zeta=\sum_{j} \eta_{j}$ is the 1 -form of Lemma 4.1. By the argument of Lemma 4.1, we see that $\not_{\tilde{\mathcal{E}} \varpi_{n}}^{+} \otimes \nabla_{n}$ commutes with the projective action $\left(\Gamma_{n}, \sigma_{n}\right)$. The analytic index is the image under the twisted Kasparov assembly map $\mu_{\sigma_{n}}: K_{\text {orb }}^{\bullet}\left(\operatorname{Sym}^{n}(\Sigma)\right) \rightarrow K_{\bullet}\left(C_{r}^{*}\left(\Gamma_{n}, \sigma_{n}\right)\right)$ of $(4.12)$,

$$
\operatorname{Ind}_{\left(\Gamma_{n}, \sigma_{n}\right)}\left(\partial_{\tilde{\mathcal{E}} \otimes_{n}}^{+} \otimes \nabla_{n}\right)=\mu_{\sigma_{n}}\left(\left[\mathcal{E}_{n}\right]\right)
$$

with the property that (§2.3 of [49])

$$
\operatorname{Ind}_{L^{2}}\left(\not_{\tilde{\mathcal{E}}^{\boxplus n}}^{+} \otimes \nabla_{n}\right)=\operatorname{tr}\left(\operatorname{Ind}_{\left(\Gamma_{n}, \sigma_{n}\right)}\left(\not_{\tilde{\mathcal{E}}^{\boxplus n}}^{+} \otimes \nabla_{n}\right)\right.
$$


A cyclic 2-cocycle on an algebra $\mathcal{R}$ is a multilinear map $t: \mathcal{R} \times \mathcal{R} \times \mathcal{R} \rightarrow$ $\mathbb{C}$ satisfying

$$
\begin{gathered}
t(a, b, c)=t(c, a, b)=t(b, c, a) \\
t(a b, c, d)-t(a, b c, d)+t(a, b, c d)-t(d a, b, c)=0 .
\end{gathered}
$$

A dense involutive subalgebra $\mathcal{R}(\Gamma, \sigma) \subset C_{r}^{*}(\Gamma, \sigma)$, which contains the twisted group ring $\mathbb{C}[\Gamma, \sigma]$ and is closed under holomorphic functional calculus is constructed in $\S 4$ of [51], as the intersection of the domains of the powers $\delta^{k}$ of the derivation $\delta=[D, \cdot]$ associated to the operator $D \delta_{\gamma}=\ell(\gamma) \delta_{\gamma}$ that multiplies group elements by the word length $\ell(\gamma)$. The Haagerup inequality for surface groups shows that group cocycles on $\Gamma$ with polynomial growth define cyclic cocycles on $\mathbb{C}[\Gamma, \sigma]$ that extend continuously to $\mathcal{R}(\Gamma, \sigma)$, see [51]. In particular, given a bounded 2-cocycle $c \in Z^{2}(\Gamma)$, one has an associated cyclic 2-cocycle $\operatorname{tr}_{c}$ on $\mathcal{R}(\Gamma, \sigma)$. This gives an additive map on $K_{0}$, which we still denote by $\operatorname{tr}_{c}$. Arguing as we did in $\$ 4$ for the cocycle defined by the magnetic field, we can identify $H^{2}(\Gamma)^{\oplus n}$ with a subspace of $H^{2}\left(\Gamma_{n}\right)$, as in Proposition 4.2. Let $c_{n}$ denote the cocycle in $H^{2}(\Gamma)^{\oplus n}$ defined by $n$ copies of the 2-cocycle $c \in H^{2}(\Gamma)$, and let $\operatorname{tr}_{c_{n}}$ be the corresponding cyclic 2-cocycle on $\mathcal{R}\left(\Gamma_{n}, \sigma_{n}\right)$. As in [17] and in $\S 3.2$ of [50], one has an associated higher twisted analytic index

$$
\begin{aligned}
& \operatorname{Ind}_{\left(c_{n}, \Gamma_{n}, \sigma_{n}\right)}\left(\not \tilde{\mathcal{E}}_{\tilde{\mathcal{E}}^{\boxplus n}}^{+} \otimes \nabla_{n}\right)=\operatorname{tr}_{c_{n}} \operatorname{Ind}_{\left(\Gamma_{n}, \sigma_{n}\right)}\left(\not_{\tilde{\mathcal{E}}^{\varpi_{n}}}^{+} \otimes \nabla_{n}\right) \\
& =\left\langle\left[\operatorname{tr}_{c_{n}}\right], \mu_{\sigma_{n}}\left(\left[\mathcal{E}_{n}\right]\right)\right\rangle=\left\langle\left[c_{n}\right],\left[\mathcal{E}_{n}\right]\right\rangle .
\end{aligned}
$$

We have the following index theorems (Theorem 1.1 of [49] and Theorem 2.2 of [50]), based on the Kawasaki index theorem on orbifold, [44], [45], see also [26], and on the higher index theorem of [17].

Proposition 6.1. Suppose given a cocycle $c \in H^{2}(\Gamma)$ as above, with $c_{n}$ the corresponding cocyle on $\Gamma_{n}$, together with a multiplier $\sigma: \Gamma \times \Gamma \rightarrow U(1)$ as in $\S 4$, determined by the closed $\Gamma$-invariant 2 -form $\omega$ of the magnetic field, and the corresponding 2-form $\omega_{n}$ on $\Sigma_{g^{\prime}}^{n}$. Then the higher twisted index (6.3) is given by

$$
\begin{aligned}
& \operatorname{tr}_{c_{n}} \operatorname{Ind}_{\left(\Gamma_{n}, \sigma_{n}\right)}\left(\not_{\tilde{\mathcal{E}}^{\bigotimes_{n}}}^{+} \otimes \nabla_{n}\right) \\
= & \frac{1}{(2 \pi)^{n} n !(\# G)^{n}} \int_{\sum_{g^{\prime}}^{n}} \hat{A}\left(\Omega_{n}\right) \operatorname{tr}\left(e^{R^{\mathcal{E}^{\prime}}{ }^{\otimes n}}\right) e^{\omega_{n}} \xi_{c_{n}},
\end{aligned}
$$

where $\xi_{c_{n}}$ is a 2-form representative of the class in $H^{2}\left(\Sigma_{g^{\prime}}\right)^{\oplus n}$ corresponding to $c_{n} \in H^{2}(\Gamma)^{\oplus n} \subset H^{2}\left(\Gamma_{n}\right)$, and $n !(\# G)^{n}=\# G_{n}$. 
The case (6.2), without the cyclic cocycle $c$, computes the range of the trace on $K$-theory, which is useful for gap labelling purposes, see [49], [51]. Here we focus on the higher version with the cyclic cocycle, as that will provide the quantization of the Hall conductance as in [51].

Lemma 6.2. Let $\mathcal{E}$ be an orbifold vector bundle over the good 2-dimensional orbifold $\Sigma=\Sigma_{g^{\prime}} / G=\mathbb{H} / \Gamma$ and $\mathcal{E}^{\prime}$ the pull back to $\Sigma_{g^{\prime}}$. Given a cocycle $c \in H^{2}(\Gamma)$ with the induced $c_{n}$ on $\Gamma_{n}=\Gamma^{n} \rtimes S_{n}$, and let $\omega$ be the 2-form determined by the magnetic field. Then the twisted higher index theorem (6.4) can be written as

$$
\begin{aligned}
& \operatorname{tr}_{c_{n}} \operatorname{Ind}_{\left(\Gamma_{n}, \sigma_{n}\right)}\left(\not \partial_{\tilde{\mathcal{E}}^{\bigotimes_{n}}}^{+} \otimes \nabla_{n}\right) \\
= & \frac{1}{(2 \pi)^{n} n !(\# G)^{n}}\left(\int_{\Sigma_{g^{\prime}}} \hat{A}(\Omega) \operatorname{tr}\left(e^{R^{\mathcal{E}^{\prime}}}\right) e^{\omega} \xi_{c}\right)^{n} .
\end{aligned}
$$

Proof. The $\hat{A}$-genus is multiplicative over products, and the form $\hat{A}\left(\Omega_{n}\right)$ on $\Sigma_{g^{\prime}}^{n}$ is the product of $n$ copies of $\hat{A}(\Omega)$ on $\Sigma_{g^{\prime}}$. The Chern character $\operatorname{ch}(\mathcal{E})=$ $\operatorname{tr}\left(e^{R^{\mathcal{E}}}\right)$ is multiplicative for external tensor products, hence $\operatorname{tr}\left(e^{R^{\mathcal{E}^{\prime}} \otimes_{n}}\right)$ on $\Sigma_{g^{\prime}}^{n}$ is also a product of $n$ copies of $\operatorname{tr}\left(e^{R^{\mathcal{E}^{\prime}}}\right)$ each depending only on one of the factors $\Sigma_{g^{\prime}}$. The form $\omega_{n}$ is by construction (see $\S 4$ ) a sum of $n$ copies of the 2 -form $\omega$, each depending only on the coordinates of one of the $\Sigma_{g^{\prime}}$ factors, hence $e^{\omega_{n}}$ is also a product. Moreover, as we have seen above, the 2 -form $\xi_{c_{n}}$ on $\Sigma_{g^{\prime}}^{n}$ is also a product of copies of a 2 -form $\xi_{c}$ on $\Sigma_{g^{\prime}}$. Thus, the integrand in (6.4) splits as a product of identical terms depending on only one of the factors.

The area 2-cocycle $c \in H^{2}(\Gamma)$ is the restriction to $\Gamma \subset \operatorname{PSL}(2, \mathbb{R})$ of the hyperbolic area $c: \operatorname{PSL}(2, \mathbb{R}) \times \operatorname{PSL}(2, \mathbb{R}) \rightarrow \mathbb{R}$, where $c\left(\gamma_{1}, \gamma_{2}\right)$ is the oriented hyperbolic area of the geodesic triangle in $\mathbb{H}$ with vertices $\left(z_{0}, \gamma_{1}^{-1} z_{0}\right.$, $\left.\gamma_{2} z_{0}\right)$, for a chosen base point $z_{0}$.

Corollary 6.3. In the case where $c \in H^{2}(\Gamma)$ is the area 2 -cocycle, the range of values of the twisted higher index theorem, while varying the choice of the orbifold vector bundle $\mathcal{E}$ on $\Sigma$ is given by

$$
\chi^{\text {orb }}\left(\operatorname{Sym}^{n}(\Sigma)\right) \mathbb{Z} \subset \mathbb{Q}
$$

where $\chi^{\text {orb }}\left(\operatorname{Sym}^{n}(\Sigma)\right)$ is the Satake orbifold Euler characteristic of $\operatorname{Sym}^{n}(\Sigma)$. 
Proof. The integral

$$
\frac{1}{2 \pi \# G} \int_{\Sigma_{g^{\prime}}} \hat{A}(\Omega) \operatorname{tr}\left(e^{R^{\mathcal{E}^{\prime}}}\right) e^{\omega} \xi_{c}
$$

is the twisted higher index theorem computed in [50]. It is shown in Corollary 3.2 of [50] that, when $c \in H^{2}(\Gamma)$ is the area 2-cocycle, this integral is given by

$$
\frac{\chi\left(\Sigma_{g^{\prime}}\right)}{\# G} \cdot \operatorname{rank}(\mathcal{E})=\chi^{\text {orb }}(\Sigma) \cdot \operatorname{rank}(\mathcal{E}) .
$$

Thus, for the area cocycle, the twisted higher index theorem (6.5) is given by

$$
\frac{\chi\left(\Sigma_{g^{\prime}}\right)^{n}}{n !(\# G)^{n}} \cdot \operatorname{rank}(\mathcal{E})^{n}=\chi^{\text {orb }}\left(\operatorname{Sym}^{n}(\Sigma)\right) \cdot \operatorname{rank}(\mathcal{E})^{n}
$$

The Hall conductance on $\Sigma$ is also described by a cyclic 2-cocycle on the twisted group algebra $\mathbb{C}[\Gamma, \sigma]$, given by

$$
\operatorname{tr}_{K}\left(f_{0}, f_{1}, f_{2}\right)=\sum_{j=1}^{g} \operatorname{tr}\left(f_{0}\left(\delta_{j}\left(f_{1}\right) \delta_{j+g}\left(f_{2}\right)-\delta_{j+g}\left(f_{1}\right) \delta_{j}\left(f_{2}\right)\right)\right),
$$

where $g$ is the genus and the $\delta_{j}$, for $j=1, \ldots, 2 g$ are derivations associated to the elements of a symplectic basis of $H^{1}(\Sigma, \mathbb{R})$. If $P_{E}$ denotes the spectral projection associated to the Fermi level, then the Hall conductance is given by

$$
\sigma_{E}=\operatorname{tr}_{K}\left(P_{E}, P_{E}, P_{E}\right)
$$

A derivation of this expression for the Hall conductance can be obtained as a quantum adiabatic limit, see [14]. As shown in Theorem 4.1 of [50], the conductance cocycle and the area cocycle are cohomologous. Since the twisted higher index theorem, seen as a pairing of cyclic cohomology and K-theory, only depends on the class of the cyclic cocycle, the range of the twisted higher index theorem also determines the possible range of values of the Hall conductance. We summarize the conclusion of this section as follows: the single particle theory on $\Sigma$ with the external magnetic field $\omega$ extends in a compatible way to a many particles model on the symmetric products $\operatorname{Sym}^{n}(\Sigma)$. In this model, the range of quantized values of the Hall conductance consists of integer multiples of the Satake orbifold Euler characteristics $\chi^{\text {orb }}\left(\operatorname{Sym}^{n}(\Sigma)\right)$. 


\section{Orbifold braid groups and anyons}

In this section we analyze what types of anyons and composite fermions one obtains within this model of fractional quantum Hall effect. These are related to a notion of "orbifold braid groups" that we introduce below.

The configuration space of $n$ (ordered) points on $\mathbb{H}$ is given by the complement of the diagonals $F(\mathbb{H}, n)=\mathbb{H}^{n} \backslash \Delta$. The configuration spaces of unordered points is defined as the quotient by the action of the symmetric group

$$
\operatorname{Conf}(\mathbb{H}, n):=\left(\mathbb{H}^{n} \backslash \Delta\right) / S_{n}=F(\mathbb{H}, n) / S_{n}
$$

These have fundamental group $\pi_{1}(\operatorname{Conf}(\mathbb{H}, n))=B_{n}$, the Artin braid group, with generators $\sigma_{i}, i=1, \ldots, n-1$ and relations $\sigma_{i} \sigma_{j}=\sigma_{j} \sigma_{i}$ for $|i-j| \geq 2$ and $\sigma_{i} \sigma_{i+1} \sigma_{i}=\sigma_{i+1} \sigma_{i} \sigma_{i+1}$, for $i=1, \ldots, n-2$. In fact, the spaces $\operatorname{Conf}(\mathbb{H}, n)$ are topologically Eilenberg-MacLane spaces $K\left(B_{n}, 1\right)$, see [23].

Given a 2-dimensional compact (topological) surface $\Sigma$, and a finite set of points $Q=\left\{x_{j}\right\}_{j=1 \ldots, m}$ on $\Sigma$, one similarly defines the configuration spaces

$$
\begin{aligned}
F(\Sigma \backslash Q, n) & =(\Sigma \backslash Q)^{n} \backslash \Delta \\
\operatorname{Conf}(\Sigma \backslash Q, n) & =F(\Sigma \backslash Q, n) / S_{n} .
\end{aligned}
$$

For $r<n$, the projections $\Pi_{n, r}\left(z_{1}, \ldots, z_{n}\right)=\left(z_{1}, \ldots, z_{r}\right)$ define locally trivial fibrations $F(\Sigma \backslash Q, n) \rightarrow F(\Sigma \backslash Q, r)$ with the fiber over $w=\left(w_{1}, \ldots, w_{r}\right)$ given by the configuration space $F\left(\Sigma \backslash\left(Q \cup\left\{w_{i}\right\}_{i=1, \ldots, r}\right), n-r\right)$. The braid group of $\Sigma \backslash Q$ on $n$ strings is given by the fundamental group

$$
B_{n}(\Sigma \backslash Q):=\pi_{1}(\operatorname{Conf}(\Sigma \backslash Q, n)) .
$$

In particular, if $\Sigma$ is a 2-dimensional orbifold and $Q \subset \Sigma$ is the set of cone points, we have corresponding braid groups

$$
B_{n}\left(\Sigma_{\text {reg }}\right)=\pi_{1}\left(\operatorname{Conf}\left(\Sigma_{\text {reg }}, n\right)\right)=\pi_{1}\left(\left(\sum_{\text {reg }}^{n} \backslash \Delta\right) / S_{n}\right) .
$$

\subsection{Orbifold braid group}

For a good 2-dimensional orbifold $\Sigma$, we can also associate to the configuration space $\operatorname{Conf}\left(\Sigma_{\mathrm{reg}}, n\right)$ an orbifold braid group, defined as

$$
B_{n}^{\text {orb }}(\Sigma):=\pi_{1}^{\text {orb }}(\operatorname{Conf}(\Sigma, n)),
$$

using the orbifold fundamental group of $\operatorname{Conf}(\Sigma, n)$. 
Proposition 7.1. The orbifold braid group of $\Sigma$ is a quotient of the ordinary braid group of $\Sigma_{\text {reg }}$ by a normal subgroup generated by powers $\gamma_{j}^{\nu_{j}}$, with $\nu_{j}$ the order of the stabilizer of the $j$-th cone point $x_{j}$ of $\Sigma$, and with $\gamma_{j}$ a loop in $\operatorname{Conf}\left(\Sigma_{\mathrm{reg}}, n\right)$ that winds around the $j$-th component of the (real) codimension two stratum of $\operatorname{Conf}(\Sigma, n)_{\text {sing }}$.

Proof. We have $\operatorname{Conf}\left(\Sigma_{\text {reg }}, n\right)=\left(\Sigma_{\text {reg }}^{n} \backslash \Delta\right) / S_{n} \subset \operatorname{Sym}^{n}(\Sigma)_{\text {reg }}$, as in Lemma 2.2 , where

$$
\begin{aligned}
& \left(\Sigma^{n} \backslash \Delta\right)_{\mathrm{reg}}=\left(\Sigma^{n} \backslash \Delta\right) \cap \Sigma_{\mathrm{reg}}^{n} \\
= & \left\{\left(z_{1}, \ldots, z_{n}\right) \in \Sigma_{\mathrm{reg}}^{n} \mid z_{i} \neq z_{j} \forall i,\right\} \\
= & \left\{\left(z_{1}, \ldots, z_{n}\right) \in \Sigma^{n} \mid z_{i} \in \Sigma_{\mathrm{reg}} \text { and } x_{i} \neq x_{j} \forall i, j\right\} \\
= & \Sigma_{\mathrm{reg}}^{n} \backslash \Delta .
\end{aligned}
$$

Moreover, $\left(\left(\sum_{\text {reg }}^{n} \backslash \Delta\right) / S_{n}\right)_{\text {reg }}=\left(\sum_{\text {reg }}^{n} \backslash \Delta\right) / S_{n}$, hence we can write the orbifold fundamental group as

$$
\pi_{1}^{\text {orb }}(\operatorname{Conf}(\Sigma, n))=\pi_{1}\left(\operatorname{Conf}\left(\Sigma_{\text {reg }}, n\right)\right) / H,
$$

where $H$ is the normal subgroup generated by elements $\gamma_{a}^{\nu_{a}}$, where the $\gamma_{a}$ are loops in $\operatorname{Conf}\left(\Sigma_{\text {reg }}, n\right)$ around a component $X_{a}$ of the singular locus $\operatorname{Conf}(\Sigma, n)_{\operatorname{sing}}$ with $\nu_{a}$ the order of the stabilizer of $X_{a}$. The singular locus $\operatorname{Conf}(\Sigma, n)_{\text {sing }}=\left(\Sigma^{n} \backslash \Delta\right)_{\text {sing }} / S_{n}$ only comes from the cone points of $\Sigma$, namely

$$
\left(\Sigma^{n} \backslash \Delta\right)_{\operatorname{sing}}=\left(\Sigma^{n}\right)_{\operatorname{sing}} \backslash \Delta,
$$

where $\left(\Sigma^{n}\right)_{\text {sing }}=\cup_{k=1}^{n} \Sigma_{\text {sing, }, k}$, with

$$
\Sigma_{\text {sing }, k}=\Sigma \times \cdots \times \Sigma \times \Sigma_{\text {sing }} \times \Sigma \cdots \times \Sigma,
$$

with a copy of $\Sigma_{\text {sing }}$ in the $k$-th factor and $\Sigma$ in all the other factors. We denote by $\Sigma_{\text {sing, }, k}\left(x_{j}\right) \subset \Sigma_{\text {sing, } k}$ the component of the (real) codimension two stratum of $\left(\Sigma^{n}\right)_{\text {sing }}$ that has a cone point $\left\{x_{j}\right\} \subset \Sigma_{\text {sing }}$ in the $k$-th factor. Thus, the components of the (real) codimension two stratum of $\left(\Sigma^{n}\right)_{\operatorname{sing}} \backslash \Delta$ are of the form $\Sigma_{\operatorname{sing}, k}\left(x_{j}\right) \backslash \Delta$. Let $\gamma_{j, k}$ be a loop in $\Sigma^{n} \backslash \Delta$ that winds around the component $\Sigma_{\text {sing, }, k}\left(x_{j}\right)$. The power $\gamma_{j, k}^{\nu_{j}}$, where $\nu_{j}$ is the order of the stabilizer of the cone point $x_{j}$ in $\Sigma$ is a generator of the subgroup $H$ of 
$\pi_{1}\left(\Sigma^{n} \backslash \Delta\right)$ such that

$$
\pi_{1}^{\text {orb }}\left(\Sigma^{n} \backslash \Delta\right)=\pi_{1}\left(\Sigma^{n} \backslash \Delta\right) / H
$$

Passing to the quotient $F(\Sigma, n)=\Sigma^{n} \backslash \Delta \rightarrow \operatorname{Conf}(\Sigma, n)=\left(\Sigma^{n} \backslash \Delta\right) / S_{n}$, the (real) codimension two stratum of the singular locus is the image of the $S_{n}$ invariant configuration of components in $F(\Sigma, n)$ given by $\Sigma_{\text {sing }}^{n}\left(x_{j}\right):=$ $\cup_{k=1}^{n} \Sigma_{\text {sing, } k}\left(x_{j}\right)$, for a given cone point $x_{j}$. We denote by $\gamma_{j}$ a loop in $\operatorname{Conf}(\Sigma, n)$ winding around $\Sigma_{\text {sing }}^{n}\left(x_{j}\right)$. The powers $\gamma_{j}^{\nu_{j}}$ generate the subgroup $H$ with

$$
\pi_{1}^{\text {orb }}(\operatorname{Conf}(\Sigma, n))=\pi_{1}\left(\operatorname{Conf}\left(\Sigma_{\mathrm{reg}}, n\right)\right) / H
$$

The braid groups of a 2-dimensional orientable (topological) surface of genus $g$ with $m$ punctures were computed explicitly in [11], [12] (see also [63], [8] for a slightly different form of the presentation). With the presentation given in [8], if $\Sigma$ has genus $g$ with a set $Q=\left\{x_{j}\right\}_{j=1, \ldots, m}$ of $m$ punctures, the braid group $B_{n}(\Sigma \backslash Q)$ has additional generators with respect to the Artin braid group $B_{n}$. Namely, the generators are given by

$$
\begin{aligned}
\sigma_{i}, & i=1, \ldots n-1 \\
a_{\ell}, & \ell=1, \ldots, g \\
b_{\ell}, & \ell=1, \ldots, g \\
c_{j}, & j=1, \ldots, m-1
\end{aligned}
$$

with relations

$$
\begin{array}{lc}
\sigma_{i} \sigma_{j}=\sigma_{j} \sigma_{i}, & \text { when }|i-j| \geq 2 \\
\sigma_{i} \sigma_{i+1} \sigma_{i}=\sigma_{i+1} \sigma_{i} \sigma_{i+1}, & i=1, \ldots, n-2
\end{array}
$$

and in the Artin braid group, and additional relations, for all $\ell$,

$$
\begin{array}{ll}
a_{\ell} \sigma_{i}=\sigma_{i} a_{\ell}, & i \neq 1 \\
b_{\ell} \sigma_{i}=\sigma_{i} b_{\ell}, & i \neq 1,
\end{array}
$$

$$
\begin{array}{cc}
\sigma_{1}^{-1} a_{\ell} \sigma_{1}^{-1} a_{\ell}=a_{\ell} \sigma_{1}^{-1} a_{\ell} \sigma_{1}^{-1} & \\
\sigma_{1}^{-1} b_{\ell} \sigma_{1}^{-1} b_{\ell}=b_{\ell} \sigma_{1}^{-1} b_{\ell} \sigma_{1}^{-1} & \\
\sigma_{1}^{-1} a_{\ell} \sigma_{1}^{-1} b_{\ell}=b_{\ell} \sigma_{1}^{-1} a_{\ell} \sigma_{1} & \\
\sigma_{1}^{-1} a_{\ell} \sigma_{1} a_{r}=a_{r} \sigma_{1}^{-1} a_{\ell} \sigma_{1} & \ell<r, \\
\sigma_{1}^{-1} b_{\ell} \sigma_{1} b_{r}=b_{r} \sigma_{1}^{-1} b_{\ell} \sigma_{1} & \ell<r, \\
\sigma_{1}^{-1} a_{\ell} \sigma_{1} b_{r}=b_{r} \sigma_{1}^{-1} a_{\ell} \sigma_{1} & \ell<r \\
\sigma_{1}^{-1} b_{\ell} \sigma_{1} a_{r}=a_{r} \sigma_{1}^{-1} b_{\ell} \sigma_{1} & \ell<r
\end{array}
$$


and for all $\ell, r$ and all $j$

$$
\begin{aligned}
c_{j} \sigma_{i} & =\sigma_{i} c_{j}, & & i \neq 1, \\
\sigma_{1}^{-1} c_{j} \sigma_{1} a_{r} & =a_{r} \sigma_{1}^{-1} c_{j} \sigma_{1} & & n>1, \\
\sigma_{1}^{-1} c_{j} \sigma_{1} b_{r} & =b_{r} \sigma_{1}^{-1} c_{j} \sigma_{1} & & n>1, \\
\sigma_{1}^{-1} c_{j} \sigma_{1} c_{k} & =c_{k} \sigma_{1}^{-1} c_{j} \sigma_{1} & & j<k, \\
\sigma_{1}^{-1} c_{j} \sigma_{1}^{-1} c_{j} & =c_{j} \sigma_{1}^{-1} c_{j} \sigma_{1}^{-1} . & &
\end{aligned}
$$

In the case without punctures, the braid group $B_{n}(\Sigma)$ has generators $\sigma_{i}$, $a_{\ell}, b_{\ell}$ as above with the same relations (7.7), (7.8), (7.9), but with (7.10) replaced by

$$
\prod_{\ell=1}^{g}\left[a_{\ell}, b_{\ell}^{-1}\right]=\sigma_{1} \sigma_{2} \cdots \sigma_{n-1}^{2} \cdots \sigma_{2} \sigma_{1} .
$$

Proposition 7.2. Let $\Sigma$ be a 2-dimensional good orbifold of genus $g$, with $m$ cone points. The orbifold braid groups $B_{n}^{\text {orb }}(\Sigma)$ has generators $\sigma_{i}, i=$ $1, \ldots, n-1, a_{\ell}, b_{\ell}, \ell=1, \ldots, g$ and $c_{j}, j=1, \ldots, m$ with relations as above, and with the additional relation

$$
\prod_{\ell=1}^{g}\left[a_{\ell}, b_{\ell}^{-1}\right] \sigma_{1}^{-1} \sigma_{2}^{-1} \cdots \sigma_{n-1}^{-2} \cdots \sigma_{2}^{-1} \sigma_{1}^{-1} c_{1} \cdots c_{m}=1
$$

and $c_{j}^{\nu_{j}}=1$, where $\nu_{j}$ is the order of the stabilizer of the cone point.

Proof. As shown in [8], the generators $a_{\ell}$ and $b_{\ell}$ correspond geometrically, in terms of a fundamental domain for $\Sigma$ given by a $4 g$-gons with pairwise identified sides marked by the generators $\alpha_{\ell}$ and $\beta_{\ell}$ of $\pi_{1}(\Sigma)$. The braid $a_{\ell}$ is a string that crosses the $\alpha_{\ell}$ sides and $b_{\ell}$ the $\beta_{\ell}$ side (with the opposite orientation), while the $c_{j}$ wind around the $j$-th puncture. The generators $\sigma_{i}$ have the usual meaning as in the Arting braid group. The relations are explained geometrically in $\S 2.2$ of [8], where it is also shown that one can equivalently introduce an additional generator $c_{m}$, as the braid that winds around the last puncture, and the additional relation (7.12). This corresponds to writing the fundamental group of the punctured surface $\Sigma \backslash Q=\Sigma_{\text {reg }}$ as

$$
\pi_{1}(\Sigma \backslash Q)=\left\langle\left\{a_{\ell}, b_{\ell}\right\}_{\ell=1, \ldots, g},\left\{c_{j}\right\}_{j=1, \ldots, m} \mid \prod_{\ell}\left[a_{\ell}, b_{\ell}^{-1}\right] c_{1} \cdots c_{m}=1\right\rangle,
$$

instead of writing it solely in terms of the generators $a_{\ell}, b_{\ell}, c_{j}$ with $j=$ $1, \ldots, m-1$. In particular, the generators $c_{j}$ provide loops in the configuration space $\operatorname{Conf}\left(\Sigma_{\text {reg }}, n\right)$ that wind around the component $\Sigma_{\text {sing }}^{n}\left(x_{j}\right)$. Thus, 
by Proposition 7.1 we obtain $B_{n}^{\text {orb }}(\Sigma)$ from $B_{n}\left(\Sigma_{\text {reg }}\right)$ by imposing the further relations $c_{j}^{\nu_{j}}=1$.

\subsection{Anyons}

Fractional-statistics particles, or anyons, have the property that, when two particles get interchanged, the wavefunction changes by a phase factor $\exp (i \pi \alpha)$, for some $\alpha \in(-1,1]$. The cases $\alpha=0$ and $\alpha=1$ correspond, respectively, to bosons and fermions. It is known [22], [38] (see also [48]) that, for 2-dimensional systems, the surface topology plays an important role in determining what type of anyons can arise. More precisely, these are classified by 1-dimensional unitary representations of the braid group of the surface. The case of the orbifold braid group is similar.

Lemma 7.3. Let $\Sigma$ be a 2 -dimensional good orbifold of genus $g>0$, with $m$ cone points. One-dimensional unitary representations $R$ of the orbifold braid group $B_{n}(\Sigma)$ have the generators $\sigma_{i}$ acting as $R\left(\sigma_{i}\right)= \pm 1$, the generators $a_{\ell}$ and $b_{\ell}$, respectively, acting as phase factors $R\left(a_{\ell}\right)=e^{2 \pi i \theta_{\ell}}$ and $R\left(b_{\ell}\right)=e^{2 \pi i \phi_{\ell}}$ and the generators $c_{j}$ acting as $R\left(c_{j}\right)=e^{2 \pi i \beta_{j} / \nu_{j}}$, where $\nu_{j}$ is the order of the stabilizer of the $j$-th cone point and the $\beta_{j}$ satisfy $\sum_{j=1}^{m} \beta_{j} / \nu_{j} \in \mathbb{Z}$.

Proof. The fact that the $\sigma_{i}$ must act like \pm 1 follows from the relation $\sigma_{1}^{-1} a_{\ell} \sigma_{1}^{-1} b_{\ell}=b_{\ell} \sigma_{1}^{-1} a_{\ell} \sigma_{1}$ in (7.9), which implies that $\sigma_{1}$ acts as \pm 1 and the relations (7.7), which imply that all the $\sigma_{i}$ must then also act in the same way. The action of the $a_{\ell}$ and $b_{\ell}$ is unconstrained by the relations, hence we get independent phase factors for each of them, while the $c_{j}$ are constrained by the relations $c_{j}^{\nu_{j}}=1$ and $(7.12)$, which implies $R\left(c_{1} \cdots c_{m}\right)=1$. These give $R\left(c_{j}\right)=e^{2 \pi i \beta_{j} / \nu_{j}}$ with $\sum_{j=1}^{m} \beta_{j} / \nu_{j} \in \mathbb{Z}$.

Remark 7.4. Since the braids $\sigma_{i}$ correspond to exchanging two particles, all the representations in Lemma 7.3 are either fermions or bosons, whenever $g>0$. The $\beta_{j}$ can be viewed as the Seifert invariants of an orbifold line bundle over $\Sigma$ that has integer orbifold Euler number (hence is an actual line bundle). See $\S 7.3$ for more details.

In the case of good 2-dimensional orbifolds of genus $g=0$ with $m$ cone points, we have the following result.

Lemma 7.5. Let $\Sigma$ be a 2-dimensional good orbifold of genus $g=0$, with $m$ cone points. Then the one-dimensional unitary representations $R$ of the 
orbifold braid group $B_{n}(\Sigma)$ have $R\left(\sigma_{i}\right)=e^{i \pi \alpha}$ and $R\left(c_{j}\right)=e^{2 \pi i \beta_{j} / \nu_{j}}$, where $\nu_{j}$ is the order of the stabilizer of the $j$-th cone point and the $\beta_{j}$ satisfy

$$
\alpha-\sum_{j=1}^{m} \frac{\beta_{j}}{\nu_{j}} \in \mathbb{Z} .
$$

Proof. The argument is exactly as in the previous lemma, except that we do not have the generators $a_{\ell}, b_{\ell}$ and the only relations are the (7.7) and

$$
\begin{array}{rlrl}
c_{j} \sigma_{i} & =\sigma_{i} c_{j}, & & i \neq 1 \\
\sigma_{1}^{-1} c_{j} \sigma_{1} c_{k} & =c_{k} \sigma_{1}^{-1} c_{j} \sigma_{1} & j<k, \\
\sigma_{1}^{-1} c_{j} \sigma_{1}^{-1} c_{j} & =c_{j} \sigma_{1}^{-1} c_{j} \sigma_{1}^{-1} & \\
\sigma_{1} \sigma_{2} \cdots \sigma_{n-1}^{2} \cdots \sigma_{2} \sigma_{1}=c_{1} \cdots c_{m} &
\end{array}
$$

and $c_{j}^{\nu_{j}}=1$. This last relation gives, as before, $R\left(c_{j}\right)=e^{2 \pi i \frac{\beta_{j}}{\nu_{j}}}$. The relations (7.7) imply that all the $\sigma_{i}$ must act by the same phase factor $R\left(\sigma_{i}\right)=$ $e^{i \pi \alpha}$ and the last displayed relation then implies that $e^{2 \pi i \alpha}=e^{2 \pi i \sum_{j=1}^{m} \beta_{j} / \nu_{j}}$, hence we obtain (7.13).

Remark 7.6. Thus, in the case of good orbifolds of genus zero, there are non-trivial anyons (that are neither fermions nor bosons) and the fractional statistics they satisfy depends on the datum of an orbifold line bundle on $\Sigma$, through the Seifert invariants $\beta_{j}$, see $\S 7.3$ below.

Moreoever, it is known that two-dimensional systems on surfaces of genus $g>0$ do admit fractional statistics arising from higher dimensional irreducible unitary representations of the braid group $B_{n}(\Sigma)$, [22], [38], provided $\alpha$ satisfies $\exp (2 \pi i(n+g-1) \alpha)=1$. We describe the analog for the orbifold braid group.

Consider the $N \times N$ matrices

$(7.14) U_{N}=\left(\begin{array}{ccccc}1 & & & & \\ & \xi_{N}^{2} & & & \\ & & \xi_{N}^{4} & & \\ & & & \ddots & \\ & & & & \xi_{N}^{2(N-1)}\end{array}\right), \quad$ with $\quad \xi_{n}=\exp (\pi i / N)$ 


$$
V_{N}=\left(\begin{array}{cccccc}
0 & 1 & 0 & \cdots & 0 & 0 \\
0 & 0 & 1 & \cdots & 0 & 0 \\
\vdots & & & & & \vdots \\
0 & 0 & 0 & \cdots & 0 & 1 \\
1 & 0 & 0 & \cdots & 0 & 0
\end{array}\right)
$$

They satisfy the commutation relation

$$
V_{N} U_{N}=\xi_{N}^{2} U_{N} V_{N}
$$

Proposition 7.7. Let $\Sigma$ be a good 2-dimensional orbifold of genus $g$ with $m$ cone points. The orbifold braid group $B_{n}^{\text {orb }}(\Sigma)$ has unitary representations of dimension $N^{g}$ with $R\left(\sigma_{i}\right)=\xi_{N}^{-1}$ and $R\left(a_{\ell}\right)=U_{N, \ell}$ and $R\left(b_{\ell}\right)=V_{N, \ell}$, where $U_{N, \ell}$ and $V_{N, \ell}$ act as $U_{N}$ and $V_{N}$, respectively, in the $\ell$-th factor of the tensor product of $g$-copies of $\mathbb{C}^{N}$ and the identity on the other factors. The generators $c_{j}$ act as $R\left(c_{j}\right)=e^{2 \pi i \beta_{j} / \nu_{j}}$, where $\nu_{j}$ is the order of the stabilizer of the $j$-th cone point and the $\beta_{j}$ satisfy the relation

$$
\frac{(g+n-1)}{N}+\sum_{j=1}^{m} \frac{\beta_{j}}{\nu_{j}} \in \mathbb{Z} .
$$

Proof. The relation $\sigma_{1}^{-1} a_{\ell} \sigma_{1}^{-1} b_{\ell}=b_{\ell} \sigma_{1}^{-1} a_{\ell} \sigma_{1}$ is now satisfied, since the lefthand-side $\xi_{N}^{2} U_{N, \ell} V_{N, \ell}$ and the right-hand-side $V_{N} U_{N}$ agree by (7.16). The rest of the relations (7.9) are also satisfied, since for $\ell \neq r U_{N, \ell}$ and $V_{N, r}$ commute. The relation $c_{j}^{\nu_{j}}=1$ is satisfied by $R\left(c_{j}\right)=e^{2 \pi i \beta_{j} / \nu_{j}}$, and the rest of the relations (7.10) are also satified. The remaining relation (7.12) for the orbifold braid groups implies

$$
\prod_{\ell=1}^{g} R\left(\left[a_{\ell}, b_{\ell}^{-1}\right]\right) \prod_{i} R\left(\sigma_{i}\right)^{-2} R\left(c_{1} \cdots c_{m}\right)=1
$$

Note that from (7.16) we have $U_{N, \ell} V_{N, \ell}^{-1}=\xi_{N}^{2} V_{N, \ell}^{-1} U_{N, \ell}$. Thus, we obtain

$$
\xi_{N}^{2 g} \cdot \xi_{N}^{2(n-1)} \cdot e^{2 \pi i \sum_{j=1}^{m} \frac{\beta_{j}}{\nu_{j}}}=1
$$

which gives $\exp \left(2 \pi i\left((g+n-1) / N+\sum_{j} \beta_{j} / \nu_{j}\right)\right)=1$, namely (7.17). 


\subsection{Orbifold line bundles and orbifold Euler numbers}

We clarify here the relation (mentioned in Remarks 7.4 and 7.6) between the anyon representations described above and the Seifert invariants of orbifold line bundles. For a complex vector bundle $\mathcal{E}$ of rank $n$ over a manifold $X$ of real dimension $2 n$, the Euler number $\chi(\mathcal{E})$ is the integral on $X$ of the Euler class $e(\mathcal{E})$. In the case of a line bundle on a 2-dimensional surface, the Euler number is the integral of the first Chern class. For an orbifold line bundle $\mathcal{L}$ on a (good) 2-dimensional orbifold $\Sigma$, the Euler number $\chi(\mathcal{L})$ is replaced by an orbifold Euler number (see [62], p.437)

$$
\chi^{\text {orb }}(\mathcal{L})=\chi(\mathcal{L})-\sum_{j=1}^{m} \frac{\beta_{j}}{\nu_{j}}
$$

where the Euler number $\chi(\mathcal{L})$ is corrected by a contribution for each cone point $x_{j}, j=1, \ldots, m$ of the orbifold. These corrections are of the form $\beta_{j} / \nu_{j}$, where $\nu_{j}$ is the order of the stabilizer $\mathbb{Z} / \nu_{j} \mathbb{Z}$ of the cone point $x_{j}$ and the $\beta_{j}$ are the Seifert invariants of the orbifold line bundles. These are obtained by considering the associated principal $U(1)$-bundle $P$ and the exact sequence

$$
1 \rightarrow \mathbb{Z} \rightarrow \pi_{1}(P) \rightarrow \Gamma \rightarrow 1
$$

where if $c_{j}$ is one of the generators of $\Gamma$ with $c_{j}^{\nu_{j}}=1$ and $\alpha$ is the generator of the fundamental group $\mathbb{Z}$ of the fiber, then the $\beta_{j}$, with $0 \leq \beta_{j} \leq \nu_{j}-1$, are defined by the relation

$$
\alpha^{\beta_{j}}=\tilde{c}_{j}
$$

where $\tilde{c}_{j}$ is a preimage of $c_{j}$ in $\pi_{1}(P)$. By the Hopf theorem, the Euler number of a line bundle on a 2-dimensional surface is a sum over zeros of a section of the line bundle, counted with multiplicity. One can then think of the orbifold Euler number (7.18) as a modification of this counting, where additional zeros are counted at the cone points, with multiplicities $\beta_{j}$, but so that each zero only contributes a fraction $1 / \nu_{j}$ of a zero at a regular point. This is consistent with the Satake orbifold Euler characteristic $\chi^{\text {orb }}(\Sigma)$, where vertices of a triangulation that are located at cone points are counted with a factor of $1 / \nu_{j}$. In fact $\chi^{\text {orb }}(\Sigma)$ is the orbifold Euler number of the orbifold tangent bundle. 


\section{Laughlin-type wave functions}

This section is more speculative in nature. It contains some observations on how one may naturally encounter some Laughlin-type functions in the geometric setting described in the previous sections. The Laughlin wave function can be regarded as a generalization of the Slater function

$$
\Psi_{\text {Slater }}\left(z_{1}, \ldots, z_{n}\right)=V\left(z_{1}, \ldots, z_{n}\right) \cdot e^{-\sum_{i=1}^{n} \frac{\left|z_{i}\right|^{2}}{4 \ell^{2}}},
$$

with the Vandermonde determinant

$$
V\left(z_{1}, \ldots, z_{n}\right)=\prod_{1 \leq i<j \leq n}\left(z_{i}-z_{j}\right)
$$

and with the magnetic length $\ell=\sqrt{\frac{\hbar c}{e B}}$. The Slater function describes noninteracting fermions in a magnetic field, for the full filling of the lowest Landau level. The Laughlin wave function takes the form

$$
\Psi_{\text {Laughlin }}\left(z_{1}, \ldots, z_{n}\right)=V\left(z_{1}, \ldots, z_{n}\right)^{p} \cdot e^{-\sum_{i=1}^{n} \frac{\left|z_{i}\right|^{2}}{4 \ell^{2}}},
$$

so that it acquires a $p$-fold zero along the diagonals $z_{i}=z_{j}$. The exponent $p$ is taken to be an odd integer, so that antisymmetry is preserved. In the case where $p$ is an even integer, one considers functions of the form

$$
\Psi_{\text {Pfaffian }}\left(z_{1}, \ldots, z_{n}\right)=\operatorname{Pfaff}\left(\frac{1}{z_{i}-z_{j}}\right) \cdot V\left(z_{1}, \ldots, z_{n}\right)^{p} \cdot e^{-\sum_{i=1}^{n} \frac{\left|z_{i}\right|^{2}}{4 \ell^{2}}} .
$$

Explicit algorithmic methods for expressing even powers of the Vandermonde determinant as combinations of Schur functions, and Laughlin wave functions as combinations of Slater functions, are discussed in [5], [61].

By analogy with the expression $\prod_{i}\left(z_{i}-z\right)^{p}$ for a vortex of vorticity $p$ centered at $z$, the Vandermonde determinant $V\left(z_{1}, \ldots, z_{n}\right)$ in the Slater wave function can be thought of as describing cyclotron motion of $n$ noninteracting fermions on the plane with magnetic field corresponding to a completely filled lower Laudau level $\nu=1$, and the corresponding powers $V\left(z_{1}, \ldots, z_{n}\right)^{p}$ in the Laughlin wave function can then be thought of similarly as vortices with vorticity $p$, see [39], [40].

We seek here some geometric interpretation of Laughlin type wave functions related to the anyon representations described in the previous section and the geometry of the orbifold symmetric products. 


\subsection{Anyon representation and orbifold vector bundle}

We associate orbifold vector bundles to the anyon representations described in the previous section.

Lemma 8.1. An $N$-dimensional anyon representations as in Proposition 7.7 determines an orbifold line bundle $\mathcal{L}$ on $\Sigma$, with pullback $\mathcal{L}^{\prime}$ to $\Sigma_{g^{\prime}}$, and an orbifold local system $V_{N}$ of (complex) rank $N$. These data in turn determine a rank $n N$ orbifold vector bundle over $\operatorname{Conf}(\Sigma, n) \subset \operatorname{Sym}^{n}(\Sigma)$ of the form $\mathcal{V}_{n, N}=\mathcal{W}_{n} \otimes V_{N}$, where $\mathcal{W}_{n}$ is the restriction to $\operatorname{Conf}(\Sigma, n)$ of the orbifold vector bundle on $\operatorname{Sym}^{n}(\Sigma)$ determined by the external Whitney sum $\mathcal{L}^{\prime \boxplus n}$ on $\Sigma_{g^{\prime}}^{n}$.

Proof. By construction, the anyon representation is representation of $\pi_{1}^{\text {orb }}(\operatorname{Conf}(\Sigma, n))$ hence it determines an orbifold local system on $\operatorname{Conf}(\Sigma, n)$, in the same way as representations of the ordinary fundamental group define local systems. The Seifert data $\beta_{j}$ of the anyon representation determine an orbifold line bundle $\mathcal{L}$ over $\Sigma$. The product $\mathcal{W}_{n} \otimes V_{N}$ is then an orbifold vector bundle over $\operatorname{Conf}(\Sigma, n)$ of complex $\operatorname{rank} n N$.

The orbifold Chern number of $\mathcal{W}_{n}$ is obtained as follows.

Lemma 8.2. Let $\mathcal{L}$ be an orbifold line bundle on the good 2-dimensional orbifold $\Sigma=\Sigma_{g^{\prime}} / G$. Let $\mathcal{L}^{\prime}$ be the pullback $G$-equivariant line bundle on $\Sigma_{g^{\prime}}$. Consider the $n$-fold external Whitney sum $\mathcal{L}^{\prime \boxplus n}$ over $\Sigma_{g^{\prime}}^{n}$, and let $\mathcal{W}_{n}$ be the corresponding orbifold vector bundle over $\operatorname{Sym}^{n}(\Sigma)$. Then the orbifold Euler number is given by

$$
\chi^{\text {orb }}\left(\mathcal{W}_{n}\right)=\frac{\chi\left(\mathcal{L}^{\prime}\right)^{n}}{n !(\# G)^{n}}=\frac{1}{n !} \chi^{\text {orb }}(\mathcal{L})^{n} .
$$

Proof. By Theorem 3.6 of [62] the orbifold Euler number $\chi^{\text {orb }}(\mathcal{L})$ of an orbifold line bundle on $\Sigma$ is related to the Euler number of a line bundle $\mathcal{L}^{\prime}$ on $\Sigma_{g^{\prime}}$ that orbifold covers $\mathcal{L}$ by

$$
\chi\left(\mathcal{L}^{\prime}\right)=\chi^{\text {orb }}(\mathcal{L}) \frac{\# G}{m},
$$

where $m$ is the number of times the circle in the fiber of the principal $U(1)$ bundle $P\left(\mathcal{L}^{\prime}\right)$ wraps around the circle in the fiber of $P(\mathcal{L})$. When $m=1$, one obtains $\chi^{\text {orb }}(\mathcal{L})=(\# G)^{-1} \chi\left(\mathcal{L}^{\prime}\right)$. The external Whitney sum is the Whitney sum $\oplus_{i=1}^{n} \pi_{i}^{*} \mathcal{L}^{\prime}$, where $\pi_{i}: \Sigma_{g^{\prime}}^{n} \rightarrow \Sigma_{g^{\prime}}$ is the projection to the $i$-th factor. The 
Euler class of a Whitney sum is the cup product of the Euler classes, hence we have $e\left(\mathcal{L}^{\prime \boxplus n}\right)=\bigwedge^{n} e\left(\mathcal{L}^{\prime}\right)$ and the Euler number is $\chi\left(\mathcal{L}^{\prime \boxplus n}\right)=\int_{\Sigma_{g^{\prime}}} e\left(\mathcal{L}^{\prime} \boxplus n\right)=$ $\chi\left(\mathcal{L}^{\prime}\right)^{n}$. Finally, the relation between the orbifold Euler number of $\mathcal{W}_{n}$ on the symmetric products $\operatorname{Sym}^{n}(\Sigma)$ and the Euler number of $\mathcal{L}^{\prime} \boxplus n$ on $\Sigma_{g^{\prime}}^{n}$ is

$$
\chi^{\text {orb }}\left(\mathcal{W}_{n}\right)=\frac{1}{\# G_{n}} \chi\left(\mathcal{L}^{\prime \otimes n}\right)=\frac{\chi\left(\mathcal{L}^{\prime}\right)^{n}}{n !(\# G)^{n}}=\frac{1}{n !} \chi^{\text {orb }}(\mathcal{L})^{n} .
$$

Local systems have torsion Chern classes, hence they do not change the differential form realizing the Euler class of $\mathcal{L}^{\prime \otimes n}$ and its integration on $\Sigma_{g^{\prime}}^{n}$.

\subsection{Mathai-Quillen formalism}

For a vector bundle $\mathcal{E}$ of (real) rank $2 n$ over a smooth manifold $X$ of (real) dimension $2 n$, the Euler class, whose integral $\chi(\mathcal{E})=\int_{X} e(\mathcal{E})$ computes the Euler number, is the pullback along the zero section of the bundle of a representative of the Thom class. Pullbacks $e_{s}(\mathcal{E})$ along other sections give the same cohomology class. By Chern-Weil theory, the Euler form can be written as the Pfaffian of the curvature $\Omega^{\mathcal{E}}$ of a hermitian connection on the bundle $\mathcal{E}$,

$$
e(\mathcal{E})=\frac{1}{(2 \pi)^{m}} \operatorname{Pfaffian}\left(\Omega^{\mathcal{E}}\right) .
$$

The Pfaffian of an antisymmetric matrix can be written in terms of the Berezin integral in fermionic coordinates

$$
\operatorname{Pfaffian}(A)=\int \mathcal{D} \xi \exp \left(\frac{1}{2} \xi^{i} A_{i j} \xi^{j}\right),
$$

hence one can write the Euler form as

$$
e(\mathcal{E})=\frac{1}{(2 \pi)^{m}} \int \mathcal{D} \xi \exp \left(\frac{1}{2} \xi^{i} \Omega_{i j}^{\mathcal{E}} \xi^{j}\right) .
$$

An explicit representative for the Thom class, which is exponentially decaying along the fibers (with normalizaed integral) and pulls back to the Euler form along the zero section is given in [52] as

$$
\Phi_{M Q}(\mathcal{E})=\frac{-\eta^{2} / 2}{(2 \pi)^{m}} \int \mathcal{D} \xi \exp \left(\frac{1}{2} \xi^{i} \Omega_{i j}^{\mathcal{E}} \xi^{j}+i \nabla \eta^{i} \xi_{i}\right),
$$

where $\eta$ are the fiber coordinates. It is shown in [52] that this is indeed a closed form representing the Thom class. The pullback along a nontrivial 
section gives

$$
e_{s}(\mathcal{E})=\frac{-s^{2} / 2}{(2 \pi)^{m}} \int \mathcal{D} \xi \exp \left(\frac{1}{2} \xi^{i} \Omega_{i j}^{\mathcal{E}} \xi^{j}+i \nabla s^{i} \xi_{i}\right) .
$$

If the section $s$ is scaled by a factor $\lambda$, in the limit of large $\lambda \rightarrow \infty$ the form $e_{s}(\mathcal{E})$ localizes on the zero set $Z_{s}=\{x \in X \mid s(x)=0\}$ of the section, hence recovering the Hopf theorem. The Mathai-Quillen formalism has found useful applications in physics, based on the observation [3] that the partition function of certain $N=1$ supersymmetric gauge theories can be written as formal functional integral analog of $\int_{X} e_{s}(\mathcal{E})$.

\subsection{Vandermonde determinants and symmetric products}

For $S=\left(s_{1}, \ldots, s_{n}\right)$, let $e_{j}(S)$ be the $j$-th elementary symmetric function in these variables, with

$$
\prod_{j=1}^{n}\left(1+t s_{j}\right)=\sum_{j=0}^{n} t^{j} e_{j}(S)
$$

Given $n$ symmetric polynomials $f_{1}, \ldots, f_{n}$, the Jacobian

$$
J\left(f_{1}, \ldots, f_{n}\right)=\operatorname{det}\left(\frac{\partial f_{i}}{\partial s_{j}}\right),
$$

while in the variables $e_{j}$, the Jacobian

$$
J_{e}\left(f_{1}, \ldots, f_{n}\right)=\operatorname{det}\left(\frac{\partial f_{i}}{\partial e_{j}}\right)
$$

is related to $J\left(f_{1}, \ldots, f_{n}\right)$ by

$$
J\left(f_{1}, \ldots, f_{n}\right)=J_{e}\left(f_{1}, \ldots, f_{n}\right) \cdot V
$$

where

$$
V\left(s_{1}, \ldots, s_{n}\right)=\prod_{1 \leq i<j \leq n}\left(s_{i}-s_{j}\right)=J\left(e_{1}, \ldots, e_{n}\right)
$$

is the Vandermonde determinant, see [46], where explicit expressions in terms of Schur functions are given for the Jacobians $J_{e}$ of complete functions and power sums. Thus, on the symmetric products $\operatorname{Sym}^{n}(\Sigma)$ we should interpret the Vandermonde determinant $V\left(s_{1}, \ldots, s_{n}\right)$ as the Jacobian of the change of local coordinates between the coordinates $\left(s_{1}, \ldots, s_{n}\right)$ of $\Sigma^{n}$ to the coordinates given by the symmetric functions $\left(e_{1}, \ldots, e_{n}\right)$. 


\subsection{Laughlin type functions from anyon representations}

Consider an $N$-dimensional anyon representation as in Proposition 7.7 and the associated orbifold vector bundle $\mathcal{V}_{n, N}$ on $\operatorname{Conf}(\Sigma, n)$, constructed as in Lemma 8.1. Let $\mathcal{L}$ be the orbifold line bundle on $\Sigma$ determined by the Seifert data of the anyon representation and $\mathcal{L}^{\prime}$ the pullback to $\Sigma_{g^{\prime}}$. Let $\underline{s}$ be a section of $\mathcal{L}^{\prime \boxplus n}$ determined by a $n$-tuple of sections of $\mathcal{L}^{\prime}$, and let $e_{\underline{s}}\left(\mathcal{L}^{\prime} \boxplus n\right)$ be the Mathai-Quillen representative of the Euler class. For the orbifold vector bundle $\mathcal{W}_{n}$, integration in the fiber direction now takes place with respect to coordinates given by the elementary symmetric functions in the original coordinates. This can be expressed in terms of the original coordinates by introducing the change of variables, as above, in the form of the Vandermonde determinant $V(\underline{s})$. Thus, we obtain an expression that has a product of $V(\underline{s})$ combined with an exponentially decaying factor in the fiber coordinates, as in the Slater wave function. When we further tensor with the local system $V_{N}$, each block of $n$-coordinates in the measure along the fiber directions acquires a factor equal to the Vandermonde determinant, while the Euler class is unchanged, hence producing a product of a power $V(\underline{s})^{N}$ of the Vandermonde determinant with an exponentially decaying factor in the $s$-coordinates, as in the Laughlin wave function. We still need to check that the power $N$ is related to the denominators of the fractions in the Hall conductance, as is the case for the Laughlin wave functions. This is satisfied in our setting, because of the relation (7.17) in the anyon representation. In fact, notice that, for the data of an orbifold vector bundle on $\Sigma$ the quantization of the Hall conductance is given, through the higher twisted index theorem, by integer multiples of Stake orbifold Euler characteristics $\chi^{\text {orb }}(\Sigma)=\chi\left(\Sigma_{g^{\prime}}\right) / \# G$, hence the denominator is the order of $G$, which is also the least common multiple of the orders $\nu_{j}$ of the stablizers of the cone points (Lemma 7.11 of [25]). The relation (7.17) then relates $N$ to $\# G$.

\subsection{Vandermonde determinants, Selberg integrals, and Euler characteristics}

The description of the Vandermonde determinant as Jacobian of the change of coordinates on symmetric products also leads to the well known probability distributions in random matrix theory

$$
\int_{\mathcal{V}_{\mathbb{F}}(n)} \exp \left(-\|y\|^{2} / 2\right) d y=\kappa \int_{\mathbb{R}^{n}} V\left(x_{1}, \ldots, x_{n}\right)^{\alpha} \exp \left(-\sum_{i} x_{i}^{2} / 2\right) d x
$$


where $\mathcal{V}_{\mathbb{F}}(n)$ is the space of $n \times n$-hermitian matrices, with $\mathbb{F}$ either the real numbers, the complex numbers or the quaternions, respectively with $\alpha=\operatorname{dim}_{\mathbb{R}} \mathbb{F} \in\{1,2,4\}$. The numerical factor $\kappa$ can be computed explicitly using Selberg integrals, in terms of Gamma functions, see [24] p.121.

Selberg integrals, and expectation values with respect to the random matrix probability distribution

$$
V\left(x_{1}, \ldots, x_{n}\right)^{\alpha} \exp \left(-\sum_{i} x_{i}^{2} / 2\right) d x
$$

play a crucial role in the Harer-Zagier computation of the orbifold Euler characteristic of the moduli spaces $\mathcal{M}_{g, n}$ of algebraic curves of genus $g$ with $n$ marked points, [36]. More recently, a parameterized Euler characteristic of $\mathcal{M}_{g, n}$ was introduced in [33]. This depends on a continuous parameter $\gamma$ and interpolates between the case of complex and real curves, respectively corresponding to $\gamma=1$ and $\gamma=1 / 2$. The parameterized Euler characteristic is expressed in [33] in terms of Jack symmetric functions and of expectation values

$$
\langle f(\lambda)\rangle=\frac{\int_{\mathbb{R}^{n}} f(\lambda)|V(\lambda)|^{2 \gamma} e^{-\frac{\gamma}{2} p_{2}(\lambda)} d \lambda}{\int_{\mathbb{R}^{n}}|V(\lambda)|^{2 \gamma} e^{-\frac{\gamma}{2} p_{2}(\lambda)} d \lambda},
$$

with $p_{2}(\lambda)=\sum_{i=1}^{n} \lambda_{i}^{2}$. More precisely, the parameterized Euler characteristic is explicitly computed in [33] in terms of Selberg integrals of the form

$$
\int_{\mathbb{R}^{n}}|V(\lambda)|^{2 \gamma} \prod_{j=1}^{n}\left(1+i \frac{\lambda_{j}}{a}\right)^{-\alpha}\left(1-i \frac{\lambda_{j}}{b}\right)^{-\beta} d \lambda
$$

which again can be computed explicitly in terms of Gamma functions.

It would be interesting to see if Laughlin type wave functions would arise in analogous computations of orbifold Euler characteristic of moduli spaces of good 2-dimensional orbifolds. Notice that a Teichmüller theory for orbifold was developed in [68], see also [16].

\section{Acknowledgments}

The first author was supported by NSF grants DMS-1007207, DMS-1201512, PHY-1205440, and DMS-1707882. The second author contributed to this project as part of his summer undergraduate research. 


\section{References}

[1] A. Adem, J. Leida, and Y. Ruan, Orbifolds and stringy topology, Cambridge 2007.

[2] P. Aluffi, Modification systems and integration in their Chow groups, Selecta Math. (N.S.) 11 (2005), no. 2, 155-202.

[3] M. Atiyah and L. Jeffrey, Topological Lagrangians and cohomology, J. Geom. Phys. 7 (1990), no. 1, 119-136.

[4] M. Atiyah and G. Segal, On equivariant Euler characteristic, Journal Geom. Phys. 6 (1989), no. 4, 671-677.

[5] C. Ballantine, Powers of the Vandermonde determinant, Schur functions, and the dimension game, DMTCS Proc. AO (2011), 87-98.

[6] P. Baum, A. Connes, and N. Higson, Classifying space for proper actions and K-theory of group $C^{*}$-algebras, Contemp. Math. 167 (1994), 241291.

[7] P. Baum, J. Brylinski, and R. MacPherson, Cohomologie équivariante délocalisée, C. R. Acad. Sci. Paris Sér. I Math. 300 (1985), 605-608.

[8] P. Bellingeri, On presentations of surface braid groups, J. Algebra 274 (2004), no. 2, 543-563.

[9] J. Bellissard, A. van Elst, and H. Schulz-Baldes, The non-commutative geometry of the quantum Hall effect, J. Math. Phys. 35 (1994), 53735451.

[10] J. Bellissard, Noncommutative geometry and quantum Hall effect, in: Proceedings of the International Congress of Mathematicians, Vol. 1, 2 (Zürich, 1994), 1238-1246, Birkhäuser, 1995.

[11] J. Birman, On braid groups, Comm. Pure Appl. Math. 22 (1969), 41-72.

[12] J. Birman, Mapping class groups and their relationship to braid groups, Comm. Pure Appl. Math. 22 (1969), 213-238.

[13] J. Bryan and J. Fulman, Orbifold Euler characteristics and the number of commuting m-tuples in the symmetric groups, Ann. Comb. 2 (1998), $1-6$.

[14] A. Carey, K. Hannabuss, V. Mathai, and P. McCann, Quantum Hall effect on the hyperbolic plane, Commun. Math. Physics 190 (1998), no. 3, 629-673. 
[15] P. A. Cherix, M. Cowling, P. Jolissaint, P. Julg, and A. Valette, Groups with the Haagerup property. Gromov's a-T-menability, Progress in Mathematics 197, Birkhäuser, 2001.

[16] S. Choi, Geometric structures on 2-orbifolds: exploration of discrete geometry, Mathematical Society of Japan, 2012.

[17] A. Connes and H. Moscovici, Cyclic cohomology, the Novikov conjecture and hyperbolic groups, Topology 29 (1990), 345-388.

[18] Y. Cornulier, Y. Stalder, and A. Valette, Proper actions of wreath products and generalizations, Trans. Amer. Math. Soc. 364 (2012), no. 6, 3159-3184.

[19] J. Cuntz, K-theoretic amenability for discrete groups, J. Reine Angew. Math. 344 (1983), 180-195.

[20] T. de Fernex, E. Lupercio, T. Nevins, and B. Uribe, Stringy Chern classes of singular varieties, Adv. Math. 208 (2007), no. 2, 597-621.

[21] L. Dixon, J. Harvey, C. Vafa, and E. Witten, Strings on orbifolds, Nuclear Physics B 261 (1985), 678-686.

[22] T. Einarsson, Fractional statistics on compact surfaces, Modern Physics Letters B 5 (1991), no. 10, 675-686.

[23] E. Fadell and L. Neuwirth, Configuration spaces, Math. Scand. 10 (1962), 111-118.

[24] J. Faraut and A. Korányi, Analysis on symmetric cones, Oxford University Press, 1994.

[25] B. Farb and D. Margalit, A primer on mapping class groups, Princeton University Press, 2012.

[26] C. Farsi, K-theoretical index theorems for good orbifolds, Proc. Am. Math. Soc. 115 (1992), 769-773.

[27] C. Farsi and C. Seaton, Algebraic structures associated to orbifold wreath products, J. K-Theory 8 (2011), no. 2, 323-338.

[28] C. Farsi and C. Seaton, Generalized twisted sectors of orbifolds, Pacific J. Math. 246 (2010), 49-74.

[29] C. Farsi and C. Seaton, Generalized orbifold Euler characteristics for general orbifolds and wreath products, Alg. Geom. Topology, 11 (2011), 523-551. 
[30] J. Fox, P. Haskell, A new proof of the K-amenability of $S U(1,1)$, in: Index theory of elliptic operators, foliations, and operator algebras (New Orleans, LA/Indianapolis, IN, 1986), 103-111, Contemp. Math. 70, Amer. Math. Soc., 1988.

[31] J. H. G. Fu, Curvature measures and Chern classes of singular varieties, J. Differential Geom. 39 (1994), no. 2, 251-280.

[32] H. Garth Dales, P. Aiena, J. Eschmeier, K. Laursen, and G. Willis, Introduction to Banach algebras, operators, and harmonic analysis, Cambridge University Press, 2003.

[33] I. P. Goulden, J. L. Harer, and D. M. Jackson, A geometric parametrization for the virtual Euler characteristics of the moduli space of real and complex algebraic curves, Trans. Amer. Math. Soc. 353 (2001), no. 11, 4405-4427.

[34] P. Green, The structure of imprimitivity algebras, J. Func. Anal. 36 (1980), 88-104.

[35] A. Grothendieck and M. Raynaud, Revêtements étales et groupe fondamental, Séminaire de Géométrie Algébrique du Bois Marie 1960-1961 (SGA I), Lecture Notes in Mathematics 224, Springer, 1971.

[36] J. Harer and D. Zagier, The Euler characteristic of the moduli space of curves, Invent. Math. 85 (1986), no. 3, 457-485.

[37] F. Hirzebruch and H. Höfer, On the Euler number of an orbifold, Math. Annalen 286 (1990), 255-260.

[38] T. D. Imbo, J. March-Russell, Exotic statistics on surfaces, Physics Letters B 252 (1990), no. 1, 84-90.

[39] L. Jacak, P. Sitko, K. Wieczorek, and A. Wójs, Quantum Hall systems: Braid groups, composite fermions and fractional charge, Oxford University Press, 2003.

[40] J. Jacak, R. Gonczarek, L. Jacak, and I. Jozwiak, Application of braid groups in 2D Hall system physics: composite Fermion structure, World Scientific, 2012.

[41] S. Kallel and W. Taamallah, The geometry and fundamental group of permutation products and fat diagonals, Canad. J. Math. 65 (2013), no. $3,575-599$. 
[42] S. Kallel and P. Salvatore, Symmetric products of two dimensional complexes, in: Recent developments in algebraic topology, Contemp. Math. 407 (2006), 147-161.

[43] G. G. Kasparov, Lorentz groups: K-theory of unitary representations and crossed products, Dokl. Akad. Nauk SSSR 275 (1984), no. 3, 541545 .

[44] T. Kawasaki, The index of elliptic operators over $V$-manifolds, Nagoya Math. J. 84 (1981), 135-157.

[45] T. Kawasaki, The signature theorem for $V$-manifolds, Topology 17 (1978), no. 1, 75-83.

[46] A. Lascuoux and P. Pragacz, Jacobians of symmetric polynomials, Annals of Combinatorica 6 (2002), 169-172.

[47] I. J. Leary, On the integral cohomology of wreath products, J. Algebra 198 (1997), no. 1, 184-239.

[48] A. Lerda, Anyons: quantum mechanics of particles with fractional statistics, Lecture Notes on Physics 14, Springer, 1992.

[49] M. Marcolli and V. Mathai, Twisted index theory on good orbifolds. I. Noncommutative Bloch theory, Commun. Contemp. Math. 1 (1999), no. 4, 553-587.

[50] M. Marcolli and V. Mathai, Twisted index theory on good orbifolds. II. Fractional quantum numbers, Comm. Math. Phys. 217 (2001), no. 1, 55-87

[51] M. Marcolli and V. Mathai, Towards the fractional quantum Hall effect: a noncommutative geometry perspective, in: Noncommutative Geometry and Number Theory, 235-261, Aspects Math., E37, Vieweg, 2006.

[52] V. Mathai and D. Quillen, Superconnections, Thom classes, and equivariant differential forms, Topology 25 (1986), no. 1, 85-110.

[53] G. Mislin, Equivariant K-homology of the classifying space for proper actions, in: Proper group actions and the Baum-Connes conjecture, pp. 1-78, Adv. Courses Math. CRM Barcelona, Birkhäuser, 2003.

[54] G. Moore and N. Read, Nonabelions in the fractional quantum Hall effect, Nucl. Phys. B 360 (1991), 362-396.

[55] M. Nakaoka, Homology of the infinite symmetric group, Ann. of Math. (2) 73 (1961), 229-257. 
[56] T. Ohmoto, Generating function of orbifold Chern classes, I: symmetric products, Math. Proc. Cambridge Phil. Soc. 144 (2008), no. 2, 423-438.

[57] T. Ohmoto, Equivariant Chern classes of singular algebraic varieties with group actions, Math. Proc. Camb. Phil. Soc. 140 (2006), 115-134.

[58] H. Oyono-Oyono, Baum-Connes conjecture and extensions, J. Reine Angew. Math. 532 (2001), 133-149.

[59] J. Packer, I. Raeburn, Twisted cross products of $C^{*}$-algebras, Math. Proc. Camb. Phil. Soc. 106 (1989), 293-311.

[60] I. Satake, The Gauss-Bonnet theorem for V-manifolds, J. Math. Soc. Japan 9 (1957), 464-492.

[61] T. Scharf, J. Y. Thibon, and B. G. Wybourne, Powers of the Vandermonde determinant and the quantum Hall effect, J. Phys. A 27 (1994), 4211-4219.

[62] P. Scott, The geometries of 3-manifolds, Bull. Lond. Math. Soc. 15 (1983), 401-487.

[63] G. P. Scott, Braid groups and the group of homeomorphisms of a surface, Proc. Cambridge Philos. Soc. 68 (1970), 605-617.

[64] G. Segal, Equivariant $K$-theory and symmetric products, unpublished manuscript.

[65] Y. Takeuchi and M. Yokoyama, The t-singular homology of orbifolds, Topology Appl. 153 (2006), 1722-1758.

[66] H. Tamanoi, Generalized orbifold Euler characteristic of symmetric products and covering spaces, Alg. Geom. Topology 3 (2003), 791-856.

[67] H. Tamanoi, Generalized orbifold Euler characteristic of symmetric products and equivariant Morava K-theory, Alg. Geom. Topology 1 (2001), 115-141.

[68] W. Thurston, The Geometry and Topology of 3-manifolds, unpublished manuscript, available at http://library.msri.org/books/gt3m.

[69] C. Vafa and E. Witten, A strong coupling test of S-duality, Nucl. Phys. B 431 (1994), 3-77.

[70] A. Valette, On the Baum-Connes assembly map for discrete groups, in: Proper group actions and the Baum-Connes conjecture, pp. 79-127, Adv. Courses Math. CRM Barcelona, Birkhäuser, 2003. 
[71] W. Wang, Equivariant K-theory, wreath products, and Heisenberg algebra, Duke Math. J. 103 (2000), no. 1, 1-23.

[72] J. Zhou, Delocalized equivariant coholomogy of symmetric products, arXiv:math/9910028.

Mathematics Department, Caltech

1200 E. California Blvd. Pasadena, CA 91125, USA

E-mail address: matilde@caltech.edu

E-mail address: kseipp@caltech.edu 